\title{
Genetic dissection of yield-related traits and mid-parent heterosis for those traits in maize (Zea mays L.)
}

Qiang $\mathrm{Yi}^{1,2+}$, Yinghong $\mathrm{Liu}^{3+}$, Xianbin Hou ${ }^{4+}$, Xiangge Zhang ${ }^{1,2}$, Hui $\mathrm{Li}^{1,2}$, Junjie Zhang ${ }^{5}$, Hanmei Liư ${ }^{5}$, Yufeng Hu ${ }^{1,2}$, Guowu $\mathrm{Yu}^{1,2}$, Yangping $\mathrm{Li}^{1,2}$, Yongbin Wang ${ }^{1,2}$ and Yubi Huang ${ }^{1,2^{*}}$

\begin{abstract}
Background: Utilization of heterosis in maize could be critical in maize breeding for boosting grain yield. However, the genetic architecture of heterosis is not fully understood. To dissect the genetic basis of yield-related traits and heterosis in maize, 301 recombinant inbred lines derived from 08 to $641 \times$ YE478 and 298 hybrids from the immortalized $\mathrm{F}_{2}\left(\mathrm{IF}_{2}\right)$ population were used to map quantitative trait loci (QTLs) for nine yield-related traits and midparent heterosis.

Results: We observed 156 QTLs, 28 pairs of loci with epistatic interaction, and 10 significant QTL $\times$ environment interactions in the inbred and hybrid mapping populations. The high heterosis in $F_{1}$ and $I_{2}$ populations for kernel weight per ear (KWPE), ear weight per ear (EWPE), and kernel number per row (KNPR) matched the high percentages of QTLs (over 50\%) for those traits exhibiting overdominance, whereas a notable predominance of loci with dominance effects (more than 70\%) was observed for traits that show low heterosis such as cob weight per ear (CWPE), rate of kernel production (RKP), ear length (EL), ear diameter (ED), cob diameter, and row number (RN). The environmentally stable QTL aRKP3-2 was identified across two mapping populations, while qKWPE9, affecting the trait mean and the mid-parent heterosis (MPH) level, explained over 18\% of phenotypic variations. Nine QTLs, qEWPE9-1, qEWPE10-1, qCWPE6, qEL8, qED2-2, aRN10-1, qKWPE9, qKWPE10-1, and aRKP4-3, accounted for over 10\% of phenotypic variation. In addition, QTL mapping identified 95 QTLs that were gathered together and integrated into 33 QTL clusters on 10 chromosomes.

Conclusions: The results revealed that (1) the inheritance of yield-related traits and MPH in the heterotic pattern improved Reid (PA) $\times$ Tem-tropic I (PB) is trait-dependent; (2) a large proportion of loci showed dominance effects, whereas overdominance also contributed to MPH for KNPR, EWPE, and KWPE; (3) marker-assisted selection for markers at genomic regions 1.09-1.11, 2.04, 3.08-3.09, and 10.04-10.05 contributed to hybrid performance per se and heterosis and were repeatedly reported in previous studies using different heterotic patterns is recommended.
\end{abstract}

Keywords: Heterosis, Grain yield, Immortalized $F_{2}$ population, Recombinant inbred lines, Quantitative trait locus

\section{Background}

Utilization of heterosis in maize is of great importance for boosting grain yield [1-4]. Hybrids in maize accounted for $65 \%$ of total maize cultivation by the late

\footnotetext{
* Correspondence: yubihuang@sohu.com

${ }^{\dagger}$ Qiang Yi, Yinghong Liu and Xianbin Hou contributed equally to this work.

'State Key Laboratory of Crop Gene Exploration and Utilization in Southwest

China, Sichuan Agricultural University, Chengdu 611130, China

${ }^{2}$ College of Agronomy, Sichuan Agricultural University, Chengdu 611130,

China

Full list of author information is available at the end of the article
}

twentieth century and had contributed to a quadrupling of annual maize production [1]. Numerous breeders have been interested in heterosis for many years; however, comprehension of the genetics implicated in heterosis for grain yield still remains elusive. Therefore, investigating and assessing genetic mechanisms of maize heterosis for grain yield would provide a paved route for understanding those phenomena and help to optimize breeding for grain yield in different heterotic groups.

(C) The Author(s). 2019 Open Access This article is distributed under the terms of the Creative Commons Attribution 4.0 International License (http://creativecommons.org/licenses/by/4.0/), which permits unrestricted use, distribution, and 
Through earlier inbred selection and experimental breeding $[5,6]$, maize breeders realized the importance of germplasm resources and heterosis. Previous studies [2, 7-13] used molecular markers, phenotypic identification, and pedigree information to divide different germplasm into different heterotic groups. The heterotic pattern Stiff Stalk Synthetic (SS) $\times$ Non-Stiff Stalk (NSS) has been widely used for maize breeding [12, 14-16]. Li and Wang [17], based on former studies, proposed five heterotic groups, namely Tangsipingtou (TSPT), Lancaster, Lancaster-like, the improved Reid (PA), and Tem-tropic I (PB). Extensive research has been focused on the TSPT $\times$ Reid heterotic pattern because it is one of the predominant heterotic patterns in northern China and has been used for the production of elite hybrids such as Zhengdan958 [18]. However, this heterotic pattern is rarely used in southwestern China, where alternative, scarcely investigated, heterotic patterns such as $\mathrm{PB} \times \mathrm{PA}$ are more suitable [17]. Therefore, the current study investigated the genetics of heterosis for yield-related traits in a $\mathrm{PB} \times \mathrm{PA}$ hybrid and the genetics of the yield-related traits per se.

Heterosis is defined as the superiority of $F_{1}$ hybrids in their performance over their parents $[19,20]$ and has been attributed to three different genetic hypotheses: dominance [21, 22], overdominance [19, 23], and/or epistasis [24, 25]. Specific loci for heterosis reported in previous studies corroborated each one of these hypotheses [26-38]. Those loci were identified using different designs such as the North Carolina design III with $F_{3}$ families or recombinant inbred lines (RILs), immortalized $\mathrm{F}_{2} \quad\left(\mathrm{IF}_{2)}\right.$ populations, or chromosome segment substitution lines (CSSLs). Those studies suggested different degrees of dominance for grain yield and inconsistency of heterotic loci across different heterotic patterns. For example, Frascaroli et al. [29] used the RILs from a cross between two elite inbred lines, B73 and H99, and three testcross populations obtained by crossing 142 RILs to each parent and $F_{1}$ found a significant correlation between heterozygosity level and phenotypic performance. Schön et al. [32] demonstrated high congruency of QTLs for heterosis of grain yield in three populations derived from the crosses of the heterotic pattern Iowa Stiff Stalk $x$ Lancaster Sure Crop and proposed that diverse alleles were fixed in each heterotic group. Tang et al. [31] identified 13 heterotic loci for yield-related traits, and Guo et al. [34] reported that the genetic basis of heterosis for grain yield of the elite maize hybrid Yuyu22 relies on the cumulative effects of dominance, overdominance, and epistasis. $\mathrm{Li}$ et al. [37] detected 38 heterotic loci for ear-related traits and suggested a genotype-dependent combination of heterotic loci in different hybrids. Notably, Wang et al. $[35,38]$ identified 169 heterotic loci associated with grain yield and yield components in two CSSL test populations.
Therefore, the results pertaining to genetics of grain yield and heterosis using crosses from well-studied heterotic patterns, such as Reid $\times$ Lancaster and TSPT $\times$ Reid, cannot be extrapolated to other heterotic patterns such $\mathrm{PA} \times \mathrm{PB}$.

In this study, in order to dissect genetic architecture of grain yield-related traits in maize and the contribution of different genetic effects to heterosis for grain yield-related traits, we evaluated 301 RILs from 08 to $641 \times$ YE478 in four environments and an $\mathrm{IF}_{2}$ population produced from those RILs under three environments. The elite line YE478, belonging to the PA heterotic group [17], is one of the hybrid foundation parents in China. The other parent, 08-641 (SAU08-641), which is derived from PB (or Tem-tropic I heterotic group) germplasm, is a typical elite inbred line widely used in southwest China since 1998. PA $\times$ PB is one of the predominant heterotic patterns in southwestern China. The main objectives of this research were (1) to detect and estimate QTLs with additive and additive-additive effects on yield-related traits using an RIL population (2) to identify QTLs with additive, dominant, and/or epistatic effect on yield-related traits using a hybrid population derived from the RIL population ( $\mathrm{IF}_{2}$ population) (3) to identify QTLs for mid-parent heterosis (MPH) using both mapping populations and (4) to identify the most suitable genomic regions associated with grain yield and $\mathrm{MPH}$ to be managed by marker-assisted selection.

\section{Results}

The performance of grain yield-related traits and heterosis across environments

The parental line Ye478 had significantly higher mean RKP and kernel number per row (KNPR) compared with those of 08-641 (Additional file 1: Figure S1). The RIL and $\mathrm{IF}_{2}$ populations showed considerable variations for yield-related traits (Fig. 1). The means of the $F_{1}$ were significantly higher than the means of any of the parental lines for ear weight per ear (EWPE), cob weight (CWPE), ear length (EL), ear diameter (ED), kernel weight per ear (KWPE), KNPR, and RKP. MPH of the hybrid Ye478 $\times 08-641$ was $<16 \%$ for ED and RKP, $43.5 \%$ for $\mathrm{EL}$, and $>70 \%$ for EWPE, KWPE, CWPE, and KNPR. Average MPH values across hybrids of the $\mathrm{IF}_{2}$ population were similar to $\mathrm{MPH}$ values for $\mathrm{F}_{1}$ (Additional file 4: Table S2). These results suggested a strong heterosis level for EWPE, KWPE, KNPR, and CWPE. The RIL and $\mathrm{IF}_{2}$ populations thus seem suitable for dissection of genetic effects involved in yield-related traits and heterosis for these traits.

In the combined analyses of variance of the RIL and $\mathrm{IF}_{2}$ populations across all environments, the sources of variation for genotype, environment, and genotype $\times$ environment interaction were highly significant $(p<0.01)$ for most yield-related traits (Table 1). The broad-sense 

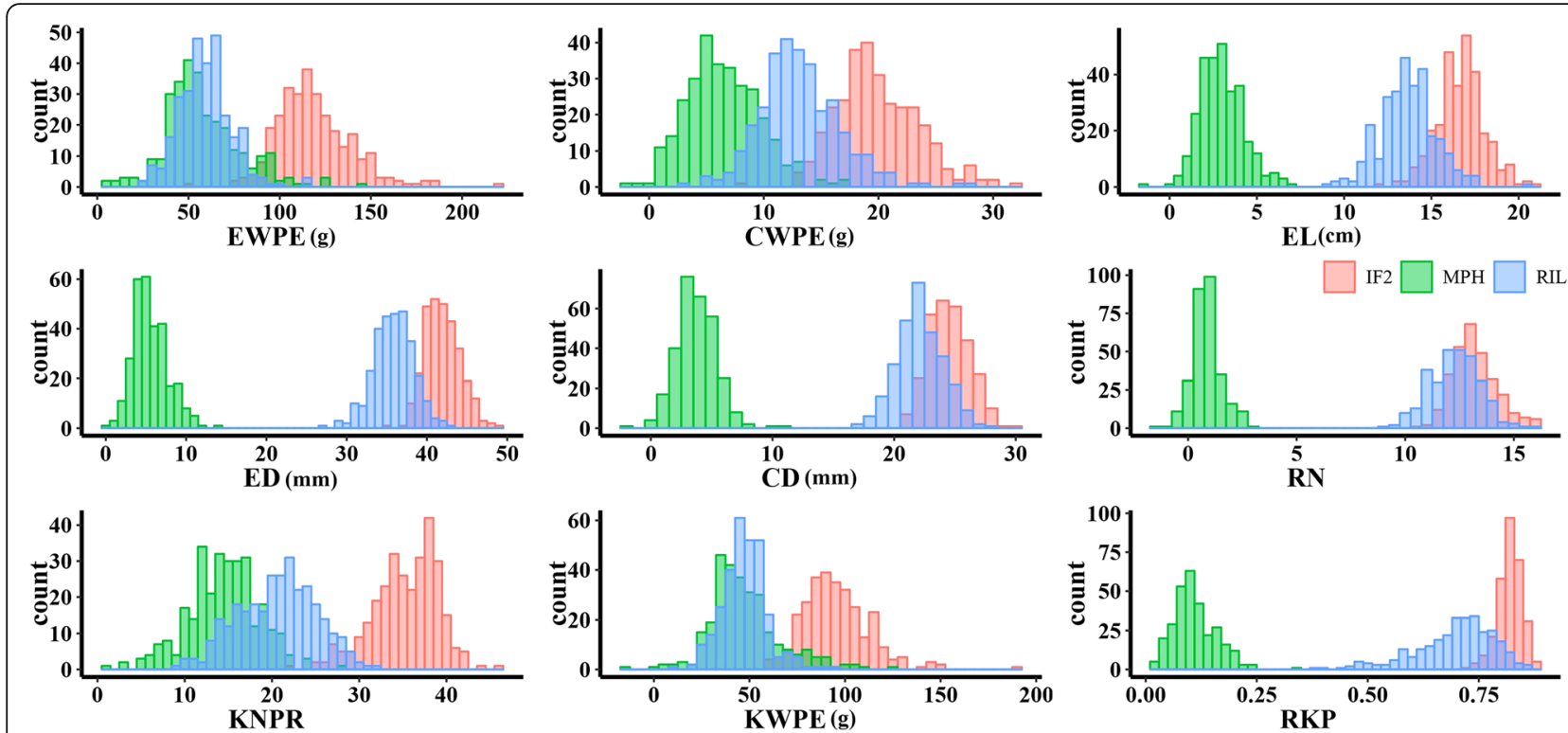

Fig. 1 The histogram for yield-related traits and mid-parent heterosis (MPH) for those traits in the RILs and the IF 2 population. EWPE, ear weight per ear; CWPE, cob weight per ear; EL, ear length; ED, ear diameter; $C D$, cob diameter; RN, row number; KNPR, kernel number per row; KWPE, kernel weight per ear; RKP, rate of kernel production

Table 1 Mean squares from the combined analyses of variance in the RILs under four environments and the $\mathrm{IF}_{2}$ population across three environments

\begin{tabular}{|c|c|c|c|c|c|c|c|}
\hline Trait $^{a}$ & Population & Gen $^{b}$ & $E v^{b}$ & $\operatorname{Rep}(E n v)^{b}$ & Gen $\times E^{2} v^{b}$ & $h^{2}(\%)^{c}$ & C. I (\%) ${ }^{d}$ \\
\hline \multirow[t]{2}{*}{ EWPE } & RIL & $1059.24^{* *}$ & $472,851.69^{* *}$ & 0.04 & $397.37^{* *}$ & 62.5 & $55.6-68.2$ \\
\hline & $\mathrm{IF}_{2}$ & $2055.73^{* *}$ & $1,080,285.18^{* *}$ & $24.60^{* *}$ & $445.28^{* *}$ & 78.3 & $74.2-81.7$ \\
\hline \multirow[t]{2}{*}{ CWPE } & RIL & $65.39^{* *}$ & $6542.52^{* *}$ & $57.93^{* *}$ & $17.89^{* *}$ & 72.6 & $67.6-76.8$ \\
\hline & $\mathrm{IF}_{2}$ & $62.46^{* *}$ & $8119.14^{* *}$ & $25.87^{* *}$ & $14.63^{* *}$ & 76.6 & $72.2-80.2$ \\
\hline \multirow[t]{2}{*}{ EL } & RIL & $18.86^{* *}$ & $293.57^{* *}$ & 0.17 & $2.65^{* *}$ & 85.9 & $83.5-87.9$ \\
\hline & $\mathrm{IF}_{2}$ & $9.66^{* *}$ & $959.04^{* *}$ & $6.97^{* *}$ & $1.33^{* *}$ & 86.2 & 83.6-88.3 \\
\hline \multirow[t]{2}{*}{ ED } & RIL & $41.48^{* *}$ & $5547.70^{* *}$ & $0.08^{* *}$ & $11.97^{* *}$ & 71.1 & $66.2-75.2$ \\
\hline & $\mathrm{IF}_{2}$ & $23.54^{* *}$ & $5304.38^{* *}$ & $8.90^{* *}$ & 6.01 & 74.5 & $69.7-78.4$ \\
\hline \multirow[t]{2}{*}{ CD } & RIL & $26.37^{* *}$ & $4715.00^{* *}$ & 7.81 & $7.55^{* *}$ & 71.3 & $66.4-75.4$ \\
\hline & $\mathrm{IF}_{2}$ & $14.27^{* *}$ & $461.95^{* *}$ & 1.6 & 4.7 & 67.1 & $60.9-72.1$ \\
\hline \multirow[t]{2}{*}{ RN } & RIL & $9.73^{* *}$ & $63.01^{* *}$ & 0.15 & $0.87^{* *}$ & 91.1 & $89.5-92.3$ \\
\hline & $\mathrm{IF}_{2}$ & $5.40^{* *}$ & $74.82^{* *}$ & 0.1 & $0.5^{* *}$ & 90.8 & $89.1-92.2$ \\
\hline \multirow[t]{2}{*}{ KNPR } & RIL & $103.85^{* *}$ & $16,419.65^{* *}$ & 8.330 & $28.42^{* *}$ & 72.6 & $67.6-76.8$ \\
\hline & $\mathrm{IF}_{2}$ & $73.69^{* *}$ & $12,860.29^{* *}$ & 1.89 & $19.65^{* *}$ & 73.3 & $68.3-77.4$ \\
\hline \multirow[t]{2}{*}{ KWPE } & RIL & $740.61^{* *}$ & $195,608.04^{* *}$ & 152.290 & $290.12^{* *}$ & 60.8 & $53.9-66.5$ \\
\hline & $\mathrm{IF}_{2}$ & $1429.09^{* *}$ & $833,331.84^{* *}$ & $28.24^{* *}$ & $315.94^{* *}$ & 77.9 & $73.8-81.3$ \\
\hline \multirow[t]{2}{*}{ RKP } & RIL & $0.05^{* *}$ & $2.62^{* *}$ & 0.00 & $0.01^{* *}$ & 77.7 & $73.8-80.9$ \\
\hline & $\mathrm{IF}_{2}$ & $0.00^{* *}$ & $0.54^{* *}$ & 0.68 & $0.00^{* *}$ & 71.3 & $66.0-75.7$ \\
\hline
\end{tabular}

${ }^{a} E W P E$ Ear weight per ear, CWPE Cob weight per ear, EL Ear length, ED Ear diameter, CD Cob diameter, RN Row number, KNPR Kernel number per ear, KWPE Kernel weight per ear, RKP the ratio of kernel production

${ }^{\mathrm{b}}$ Gen Genotype, Env Environment, Rep (Env) Replication nested within the environment, Gen $\times$ Env Genotype $\times$ environment interaction

${ }^{c} h^{2}$ Broad-sense heritability

${ }^{\mathrm{d}} C$. I the confidence interval of broad-sense heritability

"Significant at $p<0.05$ and ${ }^{* *}$ significant at $p<0.01$ 
heritability $\left(h^{2}\right)$ for these traits ranged from $60.8 \%$ for KWPE to $91.1 \%$ for RN. Heritability for EWPE and KWPE was greater in the $\mathrm{IF}_{2}$ population than in the RIL population. The correlations among most yield-related traits and among MPH values for those traits were significant (Fig. 2a; Additional file 5: Table S3). EWPE in the RIL and $\mathrm{IF}_{2}$ population was moderately correlated with CWPE, KWPE, EL, ED, and KNPR (Pearson's correlation coefficients >0.5). MPH for EWPE was moderately and positively correlated with MPH for CWPE, KWPE, EL, ED, and KNPR. The hierarchical cluster analysis based on the standardized data from the RILs classified the yield-related traits into two unrooted groups, with traits within each group being, in general, moderately to highly correlated based on Pearson's correlation coefficients (Fig. 2b).

\section{Identification of QTLs}

In the current study, 156 QTLs were found for averaged yield-related traits and MPH for those traits across all environments (Table 2; Fig. 3). Ninety-six QTLs detected across environments were also identified in at least one environment via single environment analysis (Additional file 6: Table S4). These QTLs were distributed on all chromosomes, although two QTL-concentrated regions were identified at bin 3.08-3.09 and 10.04-10.05. Each QTL explained 0.38 to $33.99 \%$ of phenotypic variance (PVE), and over half of QTLs accounted each for less than 5\% of PVE (Fig. 2c). The QTL number and the total PVE greatly differed among traits and populations (Table 2; Fig. 2d, e). Nine QTLs were detected simultaneously in the RIL and $\mathrm{IF}_{2}$ populations, while 13 QTLs detected in the $\mathrm{IF}_{2}$ population and in the MPH dataset co-localized. Only two QTLs found in the RIL population were also positioned in the same regions when QTL analyses were performed for $\mathrm{MPH}$. These results suggested that genetics involved in hybrid performance per se could be, in part, related to that implicated in MPH and it is in agreement with the moderate to high correlation coefficients between hybrid performance per se in the $\mathrm{IF}_{2}$ population and the corresponding MPH levels reported for all traits, except for RKP (Additional file 7: Table S5).
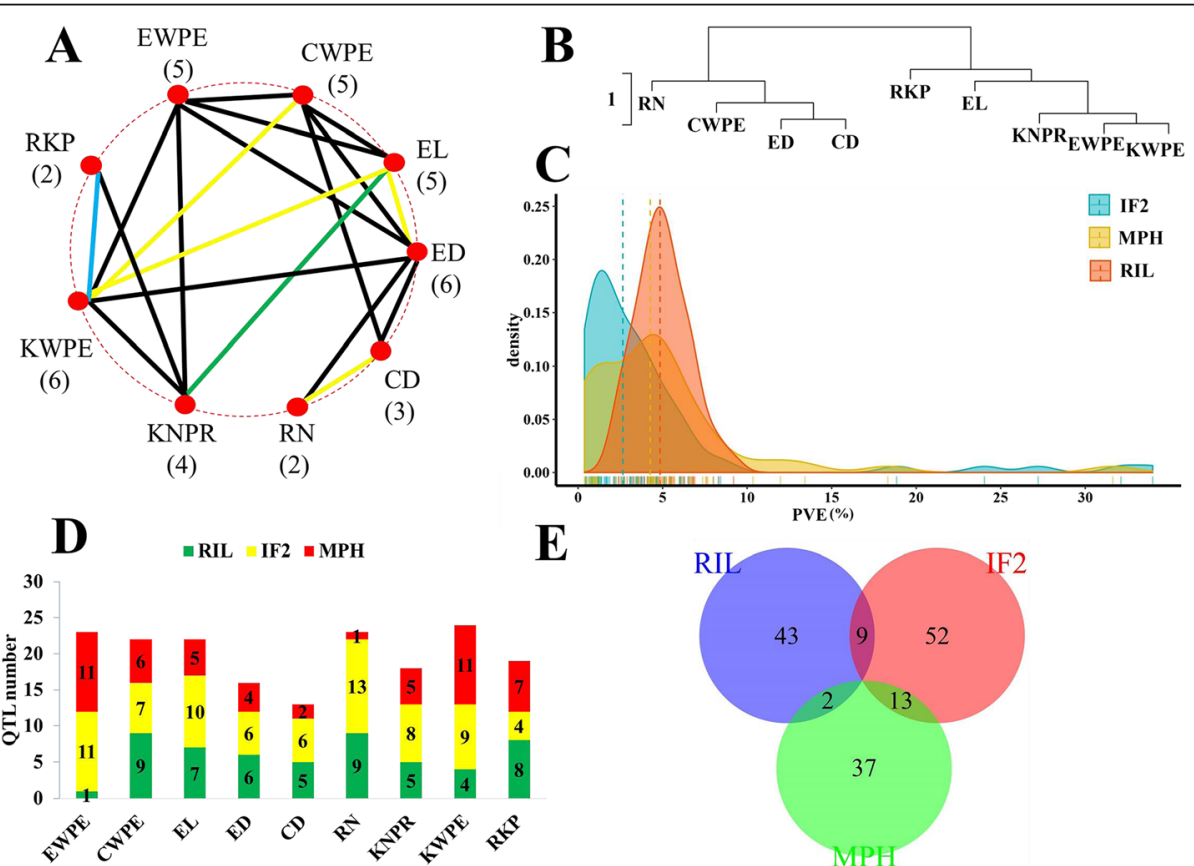

$\mathbf{E}$

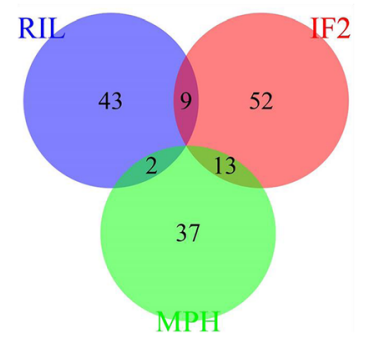

Fig. 2 The correlation relationship among yield-related traits, and mid-parent heterosis (MPH) for those traits, and QTL distribution in the different datasets. a Pearson's correlation coefficients among yield-related traits and MPH $\left(\left|r^{2}\right|>=0.5\right)$. The yellow lines indicate significant correlation found in the MPH dataset; Blue line indicates significant correlation in the RILs; green line indicates significant correlation in both the IF 2 and the MPH dataset; black lines indicate significant correlations found across the RILs, the IF 2 and the MPH dataset. The number below each trait indicates the number of the correlated traits. b Hierarchical cluster analysis ("hclust") of phenotypic traits based on the standardized data in the RILs. The short-scale indicates Euclidean distance. c Frequency distribution of QTLs with PVE (phenotypic variance explained by each QTL) detected in the RILs, IF 2 population, and the MPH dataset. The vertical and dashed lines indicate the median values. $\mathbf{d}$ The QTL numbers for nine yield-related traits identified in the RILs, IF 2 population, and the MPH dataset. e Venn diagram for the 156 QTLs detected using averaged means for yield-related traits (EWPE, ear weight per ear; CWPE, cob weight per ear; EL, ear length; ED, ear diameter; CD, cob diameter; RN, row number; KNPR, kernel number per row; KWPE, kernel weight per ear; RKP, rate of kernel production) across environments in the RILs and IF 2 populations, and the MPH dataset 
Table 2 Main features of QTLs for yield-related traits detected in the RILs, the IF 2 population, and for the mid-parental heterosis $(\mathrm{MPH})$

\begin{tabular}{|c|c|c|c|c|c|c|c|c|c|c|c|}
\hline \multirow[t]{2}{*}{ QTL } & \multirow[t]{2}{*}{$\mathrm{Chr}^{\mathrm{a}}$} & \multirow{2}{*}{$\begin{array}{l}\text { Physical } \\
\text { position }^{b}\end{array}$} & \multirow[t]{2}{*}{ Flanking marker } & \multicolumn{2}{|l|}{$\mathrm{RIL}^{\mathrm{c}}$} & \multicolumn{3}{|l|}{$\mathrm{IF}_{2}^{\mathrm{c}}$} & \multicolumn{2}{|l|}{$\mathrm{MPH}^{\mathrm{C}}$} & \multirow{2}{*}{$\begin{array}{l}\text { Detected } \\
\text { Environments }^{\mathrm{g}}\end{array}$} \\
\hline & & & & $\overline{A^{d}}$ & $\begin{array}{l}\text { PVE } \\
(\%)^{e}\end{array}$ & $\overline{A^{d}}$ & $D^{f}$ & $\begin{array}{l}\text { PVE } \\
(\%)\end{array}$ & $\overline{D^{f}}$ & $\begin{array}{l}\text { PVE } \\
(\%)\end{array}$ & \\
\hline qEWPE1-1 & 1 & $\begin{array}{l}82,577,340 / \\
87562343\end{array}$ & PZE-101090729/PZE-101094004 & & & -0.9 & 4.5 & 1.2 & & & C \\
\hline qEWPE1-2 & 1 & $\begin{array}{l}263,740,719 / \\
285391084\end{array}$ & PZE-101,213,558/SYN22772 & & & & & & 5.3 & 1.1 & C \\
\hline qEWPE2 & 2 & $\begin{array}{l}33,839,259 / \\
37868398\end{array}$ & PZE-102056295/SYN28948 & & & -1 & 7 & 2.5 & 5.8 & 1.1 & E3, C \\
\hline qEWPE3-1 & 3 & $\begin{array}{l}39,216,459 / \\
43866921\end{array}$ & PZE-103041998/SYN18260 & & & 2 & 3.9 & 1.3 & & & $\mathrm{E} 4, \mathrm{C}$ \\
\hline qEWPE3-2 & 3 & $\begin{array}{l}222,552,568 / \\
224622258\end{array}$ & SYN6986/ZM012337-0431 & & & & & & 5.4 & 0.9 & E3, C \\
\hline qEWPE4-1 & 4 & $\begin{array}{l}67,494,861 / \\
67493572\end{array}$ & PZE-104045414/PZE-104045413 & & & & & & -14.8 & 7.4 & C \\
\hline qEWPE4-2 & 4 & $\begin{array}{l}166,876,428 / \\
169511859\end{array}$ & PZE-104090796/PZE-104093153 & & & 2.3 & 3.4 & 1.2 & 3.7 & 0.9 & $\mathrm{E} 3, \mathrm{C}$ \\
\hline qEWPE6 & 6 & $\begin{array}{l}140,871,676 / \\
137826260\end{array}$ & PZE-106083588/PZE-106080884 & & & -0.2 & 5.7 & 1.7 & 6.1 & 1.3 & E3, C \\
\hline qEWPE7-1 & 7 & $\begin{array}{l}17,478,189 / \\
46213540\end{array}$ & PZE-107019133/PZE-107033682 & & & -2.1 & 5 & 1.4 & & & $\mathrm{E} 4, \mathrm{C}$ \\
\hline qEWPE7-2 & 7 & $\begin{array}{l}140,421,815 / \\
141648137\end{array}$ & PZE-107084740/PZE-107086184 & & & -1.2 & 5.6 & 1.6 & 5.6 & 1 & C \\
\hline qEWPE9-1 & 9 & $\begin{array}{l}20,233,111 / \\
24141698\end{array}$ & PZE-109019784/PZE-109023988 & & & -4.3 & 2.2 & 2.2 & & & $\mathrm{E} 3, \mathrm{C}$ \\
\hline qEWPE9-2 & 9 & $\begin{array}{l}55,513,159 / \\
93380517\end{array}$ & PZE-109037929/PZA03595.2 & & & & & & 1.7 & 31.6 & E3, C \\
\hline qEWPE9-3 & 9 & $\begin{array}{l}122,305,846 / \\
130925219\end{array}$ & PZE-109075481/PZE-109082403 & & & & & & 5.5 & 1.1 & C \\
\hline qEWPE10-1 & 10 & $\begin{array}{l}71,111,717 / \\
74412528\end{array}$ & SYN18227/PZE-110038658 & & & 17.7 & 0.6 & 32.1 & & & $\mathrm{E} 4, \mathrm{C}$ \\
\hline qEWPE10-2 & 10 & $\begin{array}{l}92,787,084 / \\
96757754\end{array}$ & PZE-110049371/PZE-110051403 & 2.3 & 6.3 & & & & & & $E 2, E 4, C$ \\
\hline qEWPE10-3 & 10 & $\begin{array}{l}124,526,091 / \\
131889929\end{array}$ & PZE-110068110/SYN17753 & & & 0.5 & 5.9 & 1.9 & 5.2 & 1 & $\mathrm{E} 4, \mathrm{C}$ \\
\hline qEWPE10-4 & 10 & $\begin{array}{l}142,189,873 / \\
146671358\end{array}$ & PZE-110095199/PZE-110104601 & & & -3.6 & 1 & 1.3 & 0.1 & 1.1 & $\mathrm{E} 3, \mathrm{C}$ \\
\hline $\begin{array}{l}\text { Total PVE } \\
\text { (\%) }\end{array}$ & & & & & 6.3 & & & 48.4 & & 48.5 & \\
\hline qCWPE1-1 & 1 & $\begin{array}{l}29,742,488 / \\
29784435\end{array}$ & PZE-101043670/PZE-101043682 & & & & & & 0.004 & 4.2 & C \\
\hline qCWPE1-2 & 1 & $\begin{array}{l}38,445,432 / \\
39742242\end{array}$ & SYN25114/PZE-101055771 & & & -0.9 & -0.4 & 2.8 & & & $E 2, E 3, C$ \\
\hline qCWPE1-3 & 1 & $\begin{array}{l}43,974,129 / \\
47135598\end{array}$ & SYN13385/SYN37775 & -0.6 & 5 & & & & & & $\mathrm{E} 3, \mathrm{C}$ \\
\hline qCWPE2-1 & 2 & $\begin{array}{l}54,088,944 / \\
54766726\end{array}$ & SYN24086/PZE-102074262 & -0.8 & 8.3 & & & & & & C \\
\hline qCWPE2-2 & 2 & $\begin{array}{l}216,546,877 / \\
217458575\end{array}$ & PZE-102173306/PZE-102175026 & 0.6 & 4.7 & & & & & & $E 3, C$ \\
\hline qCWPE2-3 & 2 & $\begin{array}{l}233,109,376 / \\
236351458\end{array}$ & PZE-102189664/PZE-102193611 & -0.4 & 2.9 & & & & & & E3, E4, C \\
\hline qCWPE3-1 & 3 & $\begin{array}{l}5,439,550 / \\
10520328\end{array}$ & SYN7905/PZE-103018221 & & & & & & 0.02 & 4.3 & $\mathrm{E} 4, \mathrm{C}$ \\
\hline
\end{tabular}


Table 2 Main features of QTLs for yield-related traits detected in the RILs, the IF 2 population, and for the mid-parental heterosis (MPH) (Continued)

\begin{tabular}{|c|c|c|c|c|c|c|c|c|c|c|c|}
\hline \multirow[t]{2}{*}{ QTL } & \multirow[t]{2}{*}{$\mathrm{Chr}^{\mathrm{a}}$} & \multirow{2}{*}{$\begin{array}{l}\text { Physical } \\
\text { position }^{\text {b }}\end{array}$} & \multirow[t]{2}{*}{ Flanking marker } & \multicolumn{2}{|l|}{$\mathrm{RIL}^{\mathrm{c}}$} & \multicolumn{3}{|l|}{$\mathrm{IF}_{2}^{\mathrm{c}}$} & \multicolumn{2}{|l|}{$\mathrm{MPH}^{\mathrm{c}}$} & \multirow{2}{*}{$\begin{array}{l}\text { Detected } \\
\text { Environments }\end{array}$} \\
\hline & & & & $\overline{A^{d}}$ & $\begin{array}{l}\text { PVE } \\
(\%)^{e}\end{array}$ & $\overline{A^{d}}$ & $D^{f}$ & $\begin{array}{l}\text { PVE } \\
(\%)\end{array}$ & $\overline{D^{f}}$ & $\begin{array}{l}\text { PVE } \\
(\%)\end{array}$ & \\
\hline qCWPE3-2 & 3 & $\begin{array}{l}215,375,486 / \\
216647518\end{array}$ & SYN33394/SYN8639 & -0.4 & 2.7 & & & & & & C \\
\hline qCWPE4-1 & 4 & $\begin{array}{l}41,851,034 / \\
56860597\end{array}$ & PZE-104033489/PZE-104041535 & & & 1 & -0.3 & 3.1 & & & $\mathrm{E} 3, \mathrm{C}$ \\
\hline qCWPE4-2 & 4 & $\begin{array}{l}142,206,530 / \\
143376752\end{array}$ & PZE-104071269/PZE-104072142 & & & -0.1 & 0.9 & 1.1 & & & $\mathrm{E} 2, \mathrm{C}$ \\
\hline qCWPE4-3 & 4 & $\begin{array}{l}166,876,428 / \\
169511859\end{array}$ & PZE-104090796/PZE-104093153 & & & & & & 0.8 & 4.3 & C \\
\hline qCWPE5 & 5 & $\begin{array}{l}167,276,024 / \\
168260189\end{array}$ & PZE-105110168/PZE-105111323 & & & -0.9 & 0.4 & 2.1 & & & $\mathrm{E} 4, \mathrm{C}$ \\
\hline qCWPE6 & 6 & $\begin{array}{l}6,268,078 / \\
24282498\end{array}$ & $\begin{array}{l}\text { PUT-163a-94,473,612-4863/PZE- } \\
106008406\end{array}$ & -0.5 & 3.5 & -3 & 0.4 & 27.2 & & & $\mathrm{E} 3, \mathrm{C}$ \\
\hline qCWPE7 & 7 & $\begin{array}{l}136,331,965 / \\
136273480\end{array}$ & PZE-107081442/PZE-107081254 & 0.4 & 2.4 & & & & & & $\mathrm{E} 2, \mathrm{C}$ \\
\hline qCWPE8 & 8 & $\begin{array}{l}19,859,565 / \\
20691990\end{array}$ & PZE-108020972/PZE-108021854 & 0.6 & 5.4 & & & & & & $\mathrm{E} 3, \mathrm{C}$ \\
\hline qCWPE9-1 & 9 & $\begin{array}{l}1,002,880 / \\
2145556\end{array}$ & PZE-109000394/PZE-109001604 & 0.5 & 4.2 & & & & & & C \\
\hline qCWPE9-2 & 9 & $\begin{array}{l}27,177,667 / \\
26944063\end{array}$ & PZE-109027216/PZE-109026940 & & & & & & 0.3 & 4.8 & $\mathrm{E} 4, \mathrm{C}$ \\
\hline qCWPE10-1 & 10 & $\begin{array}{l}96,757,754 / \\
103084814\end{array}$ & PZE-110051403/PZE-110054264 & & & 0.02 & 1 & 1.5 & & & C \\
\hline qCWPE10-2 & 10 & $\begin{array}{l}124,526,091 / \\
131889929\end{array}$ & PZE-110068110/SYN17753 & & & & & & 1.1 & 5.5 & $\mathrm{E} 4, \mathrm{C}$ \\
\hline qCWPE10-3 & 10 & $\begin{array}{l}142,189,873 / \\
146124494\end{array}$ & PZE-110095199/PZE-110103156 & & & & & & -0.04 & 5.2 & C \\
\hline qCWPE10-4 & 10 & $\begin{array}{l}148,503,131 / \\
149436870\end{array}$ & PZE-110,109,359/PZE-110111130 & & & -1 & -0.2 & 2.6 & & & C \\
\hline $\begin{array}{l}\text { Total PVE } \\
(\%)\end{array}$ & & & & & 38.9 & & & 40.5 & & 28.3 & \\
\hline qEL1-1 & 1 & $\begin{array}{l}38,445,432 / \\
42018755\end{array}$ & SYN25114/PZE-101058322 & -0.3 & 6.1 & -0.4 & -0.2 & 6.5 & & & $E 1, E 2, C$ \\
\hline qEL1-2 & 1 & $\begin{array}{l}260,149,117 / \\
263740719\end{array}$ & SYN275/PZE-101213558 & & & & & & 0.1 & 4.2 & C \\
\hline qEL2-1 & 2 & $\begin{array}{l}7,623,080 / \\
7665252\end{array}$ & PZE-102017304/PZE-102017443 & -0.3 & 4.5 & -0.4 & 0.04 & 5.5 & & & $E 1, E 2, E 3, C$ \\
\hline qEL2-2 & 2 & $\begin{array}{l}27,562,776 / \\
31293233\end{array}$ & PZE-102049280/SYN314 & -0.3 & 5.5 & & & & & & $E 1, E 3, C$ \\
\hline qEL2-3 & 2 & $\begin{array}{l}67,912,086 / \\
71361486\end{array}$ & PZE-102082146/PZE-102083803 & & & -0.2 & 0.5 & 6 & & & C \\
\hline qEL2-4 & 2 & $\begin{array}{l}148,660,513 / \\
151604997\end{array}$ & SYN34894/SYN13599 & & & & & & 0.4 & 2.2 & C \\
\hline qEL3-1 & 3 & $\begin{array}{l}24,739,373 / \\
29653189\end{array}$ & PZE-103032109/PZE-103036266 & & & 0.3 & -0.1 & 3.5 & 0.4 & 2.5 & $\mathrm{E} 3, \mathrm{C}$ \\
\hline qEL3-2 & 3 & $\begin{array}{l}175,554,472 / \\
176784418\end{array}$ & PZE-103115618/PZE-103118170 & & & 0.1 & -0.4 & 3.5 & & & $\mathrm{E} 3, \mathrm{C}$ \\
\hline qEL3-3 & 3 & $\begin{array}{l}208,785,867 / \\
210358171\end{array}$ & SYN28063/SYN20833 & -0.3 & 3.9 & -0.3 & 0.1 & 4.4 & & & $E 1, C$ \\
\hline qEL5-1 & 5 & $\begin{array}{l}135,189,300 / \\
135840044\end{array}$ & PZE-105093385/SYN32229 & 0.3 & 5.1 & & & & & & $\mathrm{E} 2, \mathrm{C}$ \\
\hline
\end{tabular}


Table 2 Main features of QTLs for yield-related traits detected in the RILs, the IF 2 population, and for the mid-parental heterosis (MPH) (Continued)

\begin{tabular}{|c|c|c|c|c|c|c|c|c|c|c|c|}
\hline \multirow[t]{2}{*}{ QTL } & \multirow[t]{2}{*}{$\mathrm{Chr}^{\mathrm{a}}$} & \multirow{2}{*}{$\begin{array}{l}\text { Physical } \\
\text { position }^{b}\end{array}$} & \multirow[t]{2}{*}{ Flanking marker } & \multicolumn{2}{|l|}{$\mathrm{RIL}^{\mathrm{c}}$} & \multicolumn{3}{|l|}{$\mathrm{IF}_{2}^{\mathrm{c}}$} & \multicolumn{2}{|l|}{$\mathrm{MPH}^{\mathrm{c}}$} & \multirow{2}{*}{$\begin{array}{l}\text { Detected } \\
\text { Environments }\end{array}$} \\
\hline & & & & $\overline{A^{d}}$ & $\begin{array}{l}\text { PVE } \\
(\%)^{e}\end{array}$ & $\overline{A^{d}}$ & $D^{f}$ & $\begin{array}{l}\text { PVE } \\
(\%)\end{array}$ & $\overline{D^{f}}$ & $\begin{array}{l}\text { PVE } \\
(\%)\end{array}$ & \\
\hline qEL5-2 & 5 & $\begin{array}{l}205,552,836 / \\
208935009\end{array}$ & PZE-105156713/PZE-105165053 & & & 0.3 & -0.1 & 3.1 & & & $E 2, C$ \\
\hline qEL5-3 & 5 & $\begin{array}{l}211,173,150 / \\
214059839\end{array}$ & SYN9389/SYN14680 & & & 0.3 & 0.2 & 3.6 & & & C \\
\hline qEL6-1 & 6 & $\begin{array}{l}140,848,748 / \\
140871676\end{array}$ & PZE-106083557/PZE-106083588 & & & 0.1 & 0.3 & 2.7 & & & C \\
\hline qEL6-2 & 6 & $\begin{array}{l}141,080,410 / \\
161454721\end{array}$ & PZE-106083873/PZE-106115356 & & & & & & 0.4 & 2.7 & $\mathrm{E} 4, \mathrm{C}$ \\
\hline qEL7 & 7 & $\begin{array}{l}136,331,965 / \\
136273480\end{array}$ & PZE-107081442/PZE-107081254 & 0.3 & 6.9 & & & & & & $E 1, E 2, C$ \\
\hline qEL8 & 8 & $\begin{array}{l}90,188,031 / \\
101409730\end{array}$ & SYN9237/PZE-108056460 & & & & & & 0.9 & 12 & $\mathrm{E} 3, \mathrm{C}$ \\
\hline qEL10-1 & 10 & $\begin{array}{l}5,761,296 / \\
6537076\end{array}$ & PZE-110007326/PZE-110008811 & & & 0.3 & 0.2 & 4.7 & & & $\mathrm{E} 4, \mathrm{C}$ \\
\hline qEL10-2 & 10 & $\begin{array}{l}102,323,080 / \\
124526091\end{array}$ & PZE-110053918/PZE-110068110 & 0.3 & 5.7 & & & & & & $\mathrm{E} 2, \mathrm{C}$ \\
\hline $\begin{array}{l}\text { Total PVE } \\
(\%)\end{array}$ & & & & & 37.6 & & & 43.6 & & 23.6 & \\
\hline qED1-1 & 1 & $\begin{array}{l}15,808,888 / \\
16371704\end{array}$ & PZE-101026314/PZE-101027182 & & & -0.4 & 0.3 & 0.9 & & & $\mathrm{E} 3, \mathrm{C}$ \\
\hline qED1-2 & 1 & $\begin{array}{l}43,974,129 / \\
47135598\end{array}$ & SYN13385/SYN37775 & -0.4 & 3.9 & & & & & & C \\
\hline qED2-1 & 2 & $\begin{array}{l}7,623,080 / \\
7665252\end{array}$ & PZE-102017304/PZE-102017443 & & & 0.5 & -0.1 & 1.2 & & & $\mathrm{E} 3, \mathrm{E} 4, \mathrm{C}$ \\
\hline aED2-2 & 2 & $\begin{array}{l}107,767,553 / \\
113810085\end{array}$ & PZE-102094429/PZE-102097841 & & & 2.2 & 0.03 & 18.8 & & & C \\
\hline qED2-3 & 2 & $\begin{array}{l}143,268,372 / \\
145993939\end{array}$ & SYN11831/PZE-102112161 & -0.4 & 4.3 & & & & & & $E 2, E 3, C$ \\
\hline qED3-1 & 3 & $\begin{array}{l}184,601,452 / \\
184674522\end{array}$ & SYN23237/SYN23245 & & & & & & 0.1 & 4.5 & C \\
\hline qED3-2 & 3 & $\begin{array}{l}211,968,759 / \\
213654405\end{array}$ & PZE-103161091/PZE-103163529 & 0.4 & 3.4 & & & & & & $E 2, C$ \\
\hline qED4 & 4 & $\begin{array}{l}166,876,428 / \\
169511859\end{array}$ & PZE-104090796/PZE-104093153 & & & & & & 0.4 & 4.3 & C \\
\hline qED6 & 6 & $\begin{array}{l}6,268,078 / \\
24282498\end{array}$ & $\begin{array}{l}\text { PUT-163a-94,473,612-4863/PZE- } \\
106008406\end{array}$ & -0.5 & 6.8 & & & & & & $E 1, E 2, E 4, C$ \\
\hline qED8-1 & 8 & $\begin{array}{l}5,605,177 / \\
6023577\end{array}$ & PZE-108005561/PZA00058.6 & 0.3 & 2.7 & & & & & & C \\
\hline qED8-2 & 8 & $\begin{array}{l}149,193,811 / \\
152752292\end{array}$ & PZE-108092173/PZE-108096683 & & & 0.4 & 0.4 & 0.8 & & & $\mathrm{E} 4, \mathrm{C}$ \\
\hline qED9-1 & 9 & $\begin{array}{l}4,010,398 / \\
4838919\end{array}$ & PZE-109003441/SYN22281 & & & 0.5 & 0.002 & 1 & & & C \\
\hline qED9-2 & 9 & $\begin{array}{l}9,501,951 / \\
16179673\end{array}$ & PZE-109008839/PZE-109015923 & 0.5 & 7.6 & & & & & & $E 1, C$ \\
\hline qED9-3 & 9 & $\begin{array}{l}55,513,159 / \\
93381704\end{array}$ & PZE-109037929/PZA03596.1 & & & -0.4 & -0.03 & 0.8 & 0.1 & 5.4 & C \\
\hline qED10 & 10 & $\begin{array}{l}88,397,317 / \\
92787084\end{array}$ & PZE-110047164/PZE-110049371 & & & & & & 0.7 & 4.5 & C \\
\hline Total PVE & & & & & 28.6 & & & 23.6 & & 18.7 & \\
\hline
\end{tabular}


Table 2 Main features of QTLs for yield-related traits detected in the RILs, the IF 2 population, and for the mid-parental heterosis (MPH) (Continued)

\begin{tabular}{|c|c|c|c|c|c|c|c|c|c|c|c|}
\hline \multirow[t]{2}{*}{ QTL } & \multirow[t]{2}{*}{$\mathrm{Chr}^{\mathrm{a}}$} & \multirow{2}{*}{$\begin{array}{l}\text { Physical } \\
\text { position }^{b}\end{array}$} & \multirow[t]{2}{*}{ Flanking marker } & \multicolumn{2}{|l|}{$\mathrm{RIL}^{\mathrm{c}}$} & \multicolumn{3}{|l|}{$\mathrm{IF}_{2}^{\mathrm{c}}$} & \multicolumn{2}{|l|}{$\mathrm{MPH}^{\mathrm{c}}$} & \multirow{2}{*}{$\begin{array}{l}\text { Detected } \\
\text { Environments }\end{array}$} \\
\hline & & & & $\overline{A^{d}}$ & $\begin{array}{l}\text { PVE } \\
(\%)^{e}\end{array}$ & $\overline{A^{d}}$ & $D^{f}$ & $\begin{array}{l}\text { PVE } \\
(\%)\end{array}$ & $\overline{D^{f}}$ & $\begin{array}{l}\text { PVE } \\
(\%)\end{array}$ & \\
\hline $9 C D 1-1$ & 1 & $\begin{array}{l}16,371,704 / \\
16602399\end{array}$ & PZE-101027182/PZE-101027807 & & & & & & 0.2 & 6.1 & C \\
\hline$q C D 1-2$ & 1 & $\begin{array}{l}38,445,432 / \\
39742242\end{array}$ & SYN25114/PZE-101055771 & & & -0.4 & -0.04 & 8.3 & & & $E 2, E 3, C$ \\
\hline$q C D 1-3$ & 1 & $\begin{array}{l}43,974,129 / \\
47135598\end{array}$ & SYN13385/SYN37775 & -0.3 & 4.3 & & & & & & $E 1, E 3, C$ \\
\hline$q C D 2$ & 2 & $\begin{array}{l}7,623,080 / \\
7665252\end{array}$ & PZE-102017304/PZE-102017443 & & & 0.3 & -0.05 & 3.7 & & & C \\
\hline$q C D 4-1$ & 4 & $\begin{array}{l}75,230,995 / \\
75633216\end{array}$ & PZE-104048874/PZE-104049163 & & & 0.4 & 0.1 & 6 & & & $\mathrm{E} 3, \mathrm{C}$ \\
\hline$q C D 4-2$ & 4 & $\begin{array}{l}99,402,352 / \\
84875360\end{array}$ & PZE-104111457/PZE-104053258 & 0.4 & 6.8 & & & & & & $E 2, C$ \\
\hline qCD4-3 & 4 & $\begin{array}{l}210,243,373 / \\
236879938\end{array}$ & PZE-104129635/PZE-104150421 & & & -0.1 & 0.5 & 6 & & & C \\
\hline qCD6 & 6 & $\begin{array}{l}60,149,277 / \\
70802055\end{array}$ & PZE-106025164/PZE-106029942 & -0.3 & 6 & & & & & & $\mathrm{E} 3, \mathrm{C}$ \\
\hline qCD7 & 7 & $\begin{array}{l}143,113,852 / \\
143294688\end{array}$ & SYN35897/PZE-107088218 & & & 0.2 & 0.2 & 3.8 & & & C \\
\hline$q C D 8-1$ & 8 & $\begin{array}{l}114,826,665 / \\
133440733\end{array}$ & SYN21795/PZE-108077809 & 0.3 & 5.2 & & & & & & $\mathrm{E} 4, \mathrm{C}$ \\
\hline$q C D 8-2$ & 8 & $\begin{array}{l}130,213,045 / \\
149193811\end{array}$ & PZE-108074750/PZE-108092173 & & & 0.3 & 0.3 & 4.1 & & & C \\
\hline qCD9 & 9 & $\begin{array}{l}1,002,880 / \\
2145556\end{array}$ & PZE-109000394/PZE-109001604 & 0.3 & 6.5 & & & & & & $E 3, C$ \\
\hline qCD10 & 10 & $\begin{array}{l}88,397,317 / \\
92787084\end{array}$ & PZE-110047164/PZE-110049371 & & & & & & 0.4 & 4.4 & $\mathrm{E} 4, \mathrm{C}$ \\
\hline $\begin{array}{l}\text { Total PVE } \\
\text { (\%) }\end{array}$ & & & & & 28.8 & & & 31.9 & & 10.5 & \\
\hline$q R N 1-1$ & 1 & $\begin{array}{l}43,974,129 / \\
47135598\end{array}$ & SYN13385/SYN37775 & -0.2 & 4.9 & & & & & & $E 1, E 2, E 4 C$ \\
\hline qRN1-2 & 1 & $\begin{array}{l}290,306,827 / \\
295888639\end{array}$ & SYN11155/PZE-101251367 & -0.2 & 3.1 & -0.2 & -0.1 & 1 & & & $\mathrm{E} 1, \mathrm{C}$ \\
\hline qRN2-1 & 2 & $\begin{array}{l}7,623,080 / \\
7665252\end{array}$ & PZE-102017304/PZE-102017443 & & & 0.2 & -0.1 & 1.4 & & & C \\
\hline$q R N 2-2$ & 2 & $\begin{array}{l}153,320,302 / \\
163564013\end{array}$ & SYN16390/SYN8399 & -0.2 & 2.1 & -0.3 & 0.1 & 1.7 & & & C \\
\hline qRN2-3 & 2 & $\begin{array}{l}226,205,828 / \\
229917830\end{array}$ & SYN8348/PZE-102186160 & & & -0.3 & 0.1 & 1.8 & & & $\mathrm{E} 4, \mathrm{C}$ \\
\hline qRN3-1 & 3 & $\begin{array}{l}1,978,736 / \\
3461364\end{array}$ & PZE-103001968/SYN25628 & & & -0.2 & -0.1 & 1.1 & & & C \\
\hline qRN3-2 & 3 & $\begin{array}{l}21,755,425 / \\
24739373\end{array}$ & PZE-103029035/PZE-103032109 & & & -0.3 & 0.1 & 1.7 & & & $E 2, E 3, E 4, C$ \\
\hline qRN3-3 & 3 & $\begin{array}{l}26,447,512 / \\
29653189\end{array}$ & PZE-103033919/PZE-103036266 & -0.3 & 5.6 & & & & & & $E 2, E 3, C$ \\
\hline qRN3-4 & 3 & $\begin{array}{l}210,358,171 / \\
211230808\end{array}$ & SYN20833/PZE-103160158 & & & 0.2 & 0.1 & 1.2 & & & $\mathrm{E} 4, \mathrm{C}$ \\
\hline qRN3-5 & 3 & $\begin{array}{l}211,968,759 / \\
213654405\end{array}$ & PZE-103161091/PZE-103163529 & 0.3 & 6.7 & & & & & & $E 1, E 2, E 4, C$ \\
\hline qRN5 & 5 & $\begin{array}{l}7,996,427 / \\
17899738\end{array}$ & PZA02029.19/PZE-105032498 & & & 0.3 & -0.04 & 2.2 & & & C \\
\hline
\end{tabular}


Table 2 Main features of QTLs for yield-related traits detected in the RILs, the IF 2 population, and for the mid-parental heterosis (MPH) (Continued)

\begin{tabular}{|c|c|c|c|c|c|c|c|c|c|c|c|}
\hline \multirow[t]{2}{*}{ QTL } & \multirow[t]{2}{*}{$\mathrm{Chr}^{\mathrm{a}}$} & \multirow{2}{*}{$\begin{array}{l}\text { Physical } \\
\text { position }^{\text {b }}\end{array}$} & \multirow[t]{2}{*}{ Flanking marker } & \multicolumn{2}{|l|}{$\mathrm{RIL}^{\mathrm{c}}$} & \multicolumn{3}{|l|}{$\mathrm{IF}_{2}^{\mathrm{C}}$} & \multicolumn{2}{|l|}{$\mathrm{MPH}^{\mathrm{c}}$} & \multirow{2}{*}{$\begin{array}{l}\text { Detected } \\
\text { Environments }\end{array}$} \\
\hline & & & & $\overline{A^{d}}$ & $\begin{array}{l}\text { PVE } \\
(\%)^{e}\end{array}$ & $\overline{A^{d}}$ & $D^{f}$ & $\begin{array}{l}\text { PVE } \\
(\%)\end{array}$ & $\overline{D^{f}}$ & $\begin{array}{l}\text { PVE } \\
(\%)\end{array}$ & \\
\hline qRN7-1 & 7 & $\begin{array}{l}775,637 / \\
1285778\end{array}$ & PZA01426.1/SYN10723 & & & 0.1 & 0.2 & 1.2 & & & $\mathrm{E} 4, \mathrm{C}$ \\
\hline qRN7-2 & 7 & $\begin{array}{l}171,743,914 / \\
172778289\end{array}$ & PZE-107130514/PZE-107132828 & -0.2 & 5.1 & -0.2 & 0.003 & 0.9 & & & $\begin{array}{l}\text { E1, E2, E3, E4, } \\
C\end{array}$ \\
\hline qRN9-1 & 9 & $\begin{array}{l}9,501,951 / \\
16179673\end{array}$ & PZE-109008839/PZE-109015923 & 0.3 & 6.2 & & & & & & $E 1, E 2, E 4, C$ \\
\hline qRN9-2 & 9 & $\begin{array}{l}34,632,675 / \\
45578052\end{array}$ & PZB00235.1/PZE-109035290 & & & -0.4 & -0.1 & 3.3 & & & $E 2, E 4, C$ \\
\hline qRN9-3 & 9 & $\begin{array}{l}97,255,443 / \\
98356545\end{array}$ & PZE-109056596/PZE-109057210 & -0.3 & 9.2 & & & & & & $\begin{array}{l}\mathrm{E1}, \mathrm{E2}, \mathrm{E3}, \mathrm{E} 4, \\
\mathrm{C}\end{array}$ \\
\hline qRN10-1 & 10 & $\begin{array}{l}74,412,528 / \\
71111717\end{array}$ & PZE-110038658/SYN18227 & & & 1 & 0.1 & 24 & & & $\mathrm{E} 3, \mathrm{C}$ \\
\hline qRN10-2 & 10 & $\begin{array}{l}37,810,598 / \\
82232426\end{array}$ & PZE-110027837/PZE-110043216 & 0.2 & 2.4 & 0.4 & 0.1 & 3.4 & & & $E 2, E 3, C$ \\
\hline qRN10-3 & 10 & $\begin{array}{l}103,422,421 / \\
102323080\end{array}$ & PZE-110054411/PZE-110053918 & & & & & & -0.1 & 6.6 & $\mathrm{E} 3, \mathrm{C}$ \\
\hline $\begin{array}{l}\text { Total PVE } \\
\text { (\%) }\end{array}$ & & & & & 45.2 & & & 44.9 & & 6.6 & \\
\hline qKNPR1-1 & 1 & $\begin{array}{l}9,434,924 / \\
11488064\end{array}$ & SYN14143/PZE-101019726 & & & -0.8 & 0.1 & 4.3 & & & C \\
\hline qKNPR1-2 & 1 & $\begin{array}{l}217,114,855 / \\
220711263\end{array}$ & PZE-101173330/SYN2411 & & & -0.7 & 0.6 & 5.1 & & & $\mathrm{E} 3, \mathrm{C}$ \\
\hline qKNPR2-1 & 2 & $\begin{array}{l}65,134,549 / \\
63665753\end{array}$ & PZE-102080979/PZE-102080069 & & & -0.3 & 1.3 & 6.3 & & & $\mathrm{E} 4, \mathrm{C}$ \\
\hline qKNPR2-2 & 2 & $\begin{array}{l}177,239,688 / \\
178977489\end{array}$ & PZE-102127480/PZE-102129070 & & & & & & 1.1 & 4.4 & $\mathrm{E} 2, \mathrm{C}$ \\
\hline qKNPR3-1 & 3 & $\begin{array}{l}211,968,759 / \\
213654405\end{array}$ & PZE-103161091/PZE-103163529 & 0.9 & 8 & & & & & & $E 2, E 4, C$ \\
\hline qKNPR3-2 & 3 & $\begin{array}{l}224,622,258 / \\
227274031\end{array}$ & ZM012337-0431/PZE-103182712 & & & 0.5 & 1 & 5.1 & & & C \\
\hline qKNPR4-1 & 4 & $\begin{array}{l}7,155,405 / \\
10656171\end{array}$ & PZE-104010113/PZE-104012412 & & & -0.6 & 0.6 & 3.3 & & & C \\
\hline qKNPR4-2 & 4 & $\begin{array}{l}35,272,230 / \\
35542248\end{array}$ & PZE-104029222/PZE-104029384 & & & & & & 1 & 5.2 & $\mathrm{E} 4, \mathrm{C}$ \\
\hline qKNPR5-1 & 5 & $\begin{array}{l}135,840,044 / \\
135189300\end{array}$ & SYN32229/PZE-105093385 & 0.7 & 4.7 & & & & & & $\mathrm{E} 4, \mathrm{C}$ \\
\hline qKNPR5-2 & 5 & $\begin{array}{l}167,276,024 / \\
168260189\end{array}$ & PZE-105110168/PZE-105111323 & & & & & & -0.1 & 7.5 & C \\
\hline qKNPR6-1 & 6 & $\begin{array}{l}105,500,541 / \\
106069077\end{array}$ & PZE-106055082/PZE-106055781 & -0.6 & 3.2 & & & & & & C \\
\hline qKNPR6-2 & 6 & $\begin{array}{l}140,871,676 / \\
137826260\end{array}$ & PZE-106083588/PZE-106080884 & & & 0.1 & 1.1 & 3.9 & 1.1 & 4.2 & $\mathrm{E} 3, \mathrm{E} 4, \mathrm{C}$ \\
\hline qKNPR7-1 & 7 & $\begin{array}{l}140,421,815 / \\
141648137\end{array}$ & PZE-107084740/PZE-107086184 & & & -0.1 & 1 & 3.1 & & & $\mathrm{E} 3, \mathrm{C}$ \\
\hline qKNPR7-2 & 7 & $\begin{array}{l}149,763,210 / \\
149725744\end{array}$ & PZE-107094398/PZE-107094385 & -0.7 & 4.6 & & & & & & C \\
\hline qKNPR9 & 9 & $\begin{array}{l}101,477,167 / \\
105064711\end{array}$ & PZB01899.2/SYN37647 & & & & & & 0.8 & 3.6 & C \\
\hline aKNPR10-1 & 10 & $\begin{array}{l}58,452,435 / \\
82232426\end{array}$ & PZE-110025994/PZE-110043216 & & & 0.9 & 0.4 & 6.1 & & & $\mathrm{E} 4, \mathrm{C}$ \\
\hline
\end{tabular}


Table 2 Main features of QTLs for yield-related traits detected in the RILs, the IF 2 population, and for the mid-parental heterosis (MPH) (Continued)

\begin{tabular}{|c|c|c|c|c|c|c|c|c|c|c|c|}
\hline \multirow[t]{2}{*}{ QTL } & \multirow[t]{2}{*}{$\mathrm{Chr}^{\mathrm{a}}$} & \multirow{2}{*}{$\begin{array}{l}\text { Physical } \\
\text { position }^{b}\end{array}$} & \multirow[t]{2}{*}{ Flanking marker } & \multicolumn{2}{|l|}{$\mathrm{RIL}^{\mathrm{c}}$} & \multicolumn{3}{|l|}{$\mathrm{IF}_{2}^{\mathrm{c}}$} & \multicolumn{2}{|l|}{$\mathrm{MPH}^{\mathrm{C}}$} & \multirow{2}{*}{$\begin{array}{l}\text { Detected } \\
\text { Environments }\end{array}$} \\
\hline & & & & $\overline{A^{d}}$ & $\begin{array}{l}\text { PVE } \\
(\%)^{e}\end{array}$ & $\overline{A^{d}}$ & $D^{f}$ & $\begin{array}{l}\text { PVE } \\
(\%)\end{array}$ & $\overline{D^{f}}$ & $\begin{array}{l}\text { PVE } \\
(\%)\end{array}$ & \\
\hline aKNPR10-2 & 10 & $\begin{array}{l}92,787,084 / \\
96757754\end{array}$ & PZE-110049371/PZE-110051403 & 0.8 & 5.5 & & & & & & C \\
\hline $\begin{array}{l}\text { Total PVE } \\
(\%)\end{array}$ & & & & & 26 & & & 37.1 & & 25 & \\
\hline qKWPE1-1 & 1 & $\begin{array}{l}43,974,129 / \\
47135598\end{array}$ & SYN13385/SYN37775 & & & -1.3 & 3.6 & 0.4 & & & C \\
\hline qKWPE1-2 & 1 & $\begin{array}{l}73,736,703 / \\
77488489\end{array}$ & PZE-101084850/PZE-101087156 & & & -1.3 & 3.9 & 0.4 & & & C \\
\hline qKWPE1-3 & 1 & $\begin{array}{l}232,527,769 / \\
245035242\end{array}$ & PZE-101187496/PZE-101196838 & -1.9 & 6.6 & & & & & & $\mathrm{E} 1, \mathrm{C}$ \\
\hline qKWPE1-4 & 1 & $\begin{array}{l}263,740,719 / \\
285391084\end{array}$ & PZE-101213558/SYN22772 & & & & & & 5.3 & 0.8 & C \\
\hline qKWPE2-1 & 2 & $\begin{array}{l}37,868,398 / \\
38140456\end{array}$ & SYN28948/PZE-102059924 & & & -0.4 & 5.7 & 0.8 & & & $\mathrm{E} 3, \mathrm{C}$ \\
\hline qKWPE2-2 & 2 & $\begin{array}{l}43,372,951 / \\
47158891\end{array}$ & PZE-102065424/PZA02450.1 & & & & & & -16.6 & 8 & $\mathrm{E} 3, \mathrm{C}$ \\
\hline qKWPE2-3 & 2 & $\begin{array}{l}188,055,798 / \\
190159942\end{array}$ & SYN19995/SYN5428 & & & & & & 1.7 & 3.2 & C \\
\hline qKWPE2-4 & 2 & $\begin{array}{l}192,581,253 / \\
192700757\end{array}$ & PZE-102145606/PZE-102145703 & & & & & & 1.2 & 5 & C \\
\hline qKWPE2-5 & 2 & $\begin{array}{l}201,971,890 / \\
209300839\end{array}$ & PZE-102154251/SYN7501 & & & -3.3 & 1.7 & 0.5 & & & C \\
\hline qKWPE3-1 & 3 & $\begin{array}{l}211,968,759 / \\
213654405\end{array}$ & PZE-103161091/PZE-103163529 & 1.7 & 5.5 & & & & & & $E 1, E 2, C$ \\
\hline qKWPE3-2 & 3 & $\begin{array}{l}222,552,568 / \\
224622258\end{array}$ & SYN6986/ZM012337-0431 & & & & & & 3.9 & 0.5 & C \\
\hline qKWPE4 & 4 & $\begin{array}{l}166,876,428 / \\
169511859\end{array}$ & PZE-104090796/PZE-104093153 & & & & & & 2.5 & 0.4 & C \\
\hline qKWPE6-1 & 6 & $\begin{array}{l}140,871,676 / \\
137826260\end{array}$ & PZE-106083588/PZE-106080884 & & & -0.4 & 4.9 & 0.6 & 4.7 & 0.7 & $E 1, E 3, E 4, C$ \\
\hline qKWPE6-2 & 6 & $\begin{array}{l}142,423,964 / \\
152749874\end{array}$ & PZE-105096701/PZE-105101729 & 1.4 & 3.7 & & & & & & C \\
\hline qKWPE7-1 & 7 & $\begin{array}{l}17,478,189 / \\
46213540\end{array}$ & PZE-107019133/PZE-107033682 & & & -1.8 & 5.8 & 0.8 & 5 & 0.8 & $E 2, E 4, C$ \\
\hline qKWPE7-2 & 7 & $\begin{array}{l}140,421,815 / \\
141648137\end{array}$ & PZE-107084740/PZE-107086184 & & & & & & 3.8 & 0.5 & C \\
\hline qKWPE7-3 & 7 & $\begin{array}{l}144,000,432 / \\
144753122\end{array}$ & PZE-107088998/PZE-107089819 & & & -1.9 & 3.9 & 0.4 & & & $E 3, C$ \\
\hline QKWPE9 & 9 & $\begin{array}{l}55,513,159 / \\
93380517\end{array}$ & PZE-109037929/PZA03595.2 & & & -26.2 & 1.8 & 34 & 1.5 & 18.3 & $\mathrm{E} 2, \mathrm{E} 4, \mathrm{C}$ \\
\hline qKWPE10-1 & 10 & $\begin{array}{l}23,214,551 / \\
25558345\end{array}$ & PZE-110019199/PZE-110020162 & & & & & & -21.3 & 13.4 & C \\
\hline aKWPE10-2 & 10 & $\begin{array}{l}124,526,091 / \\
131889929\end{array}$ & PZE-110068110/SYN17753 & & & 2.4 & 3.9 & 0.7 & & & $\mathrm{E} 4, \mathrm{C}$ \\
\hline 9KWPE10-3 & 10 & $\begin{array}{l}131,104,195 / \\
134219127\end{array}$ & PZE-110074914/PZE-110079903 & 1.6 & 4.4 & & & & & & C \\
\hline $\begin{array}{l}\text { Total PVE } \\
(\%)\end{array}$ & & & & & 20.1 & & & 38.8 & & 51.6 & \\
\hline qRKP1-1 & 1 & $\begin{array}{l}223,825,525 / \\
229541701\end{array}$ & SYN31271/PZE-101184757 & -0.01 & 4.7 & -0.004 & 0.004 & 3.6 & & & E1, E3, C \\
\hline
\end{tabular}


Table 2 Main features of QTLs for yield-related traits detected in the RILs, the $\mathrm{IF}_{2}$ population, and for the mid-parental heterosis $(\mathrm{MPH})$ (Continued)

\begin{tabular}{|c|c|c|c|c|c|c|c|c|c|c|c|}
\hline \multirow[t]{2}{*}{ QTL } & \multirow[t]{2}{*}{$\mathrm{Chr}^{\mathrm{a}}$} & \multirow{2}{*}{$\begin{array}{l}\text { Physical } \\
\text { position }^{\mathrm{b}}\end{array}$} & \multirow[t]{2}{*}{ Flanking marker } & \multicolumn{2}{|l|}{$\mathrm{RIL}^{\mathrm{c}}$} & \multicolumn{3}{|l|}{$\mathrm{IF}_{2}^{\mathrm{C}}$} & \multicolumn{2}{|l|}{$\mathrm{MPH}^{\mathrm{c}}$} & \multirow{2}{*}{$\begin{array}{l}\text { Detected } \\
\text { Environments }^{g}\end{array}$} \\
\hline & & & & $\overline{A^{d}}$ & $\begin{array}{l}\text { PVE } \\
(\%)^{\mathrm{e}}\end{array}$ & $\mathrm{A}^{\mathrm{d}}$ & $D^{f}$ & $\begin{array}{l}\text { PVE } \\
(\%)\end{array}$ & $\overline{D^{f}}$ & $\begin{array}{l}\text { PVE } \\
(\%)\end{array}$ & \\
\hline qRKP1-2 & 1 & $\begin{array}{l}232,527,769 / \\
245035242\end{array}$ & PZE-101187496/PZE-101196838 & & & & & & -0.003 & 7.7 & $C$ \\
\hline qRKP3-1 & 3 & $\begin{array}{l}184,674,522 / \\
191932135\end{array}$ & SYN23245/PZE-103136534 & 0.01 & 4.8 & & & & -0.003 & 5.7 & $E 1, E 2, E 4, C$ \\
\hline qRKP3-2 & 3 & $\begin{array}{l}211,230,808 / \\
213654405\end{array}$ & PZE-103160158/PZE-103163529 & 0.01 & 4.3 & & & & 0.01 & 6.7 & $E 2, E 3, E 4, C$ \\
\hline qRKP3-3 & 3 & $\begin{array}{l}227,274,031 / \\
227827542\end{array}$ & PZE-103182712/PZE-103183391 & 0.01 & 3.6 & & & & & & C \\
\hline qRKP4-1 & 4 & $\begin{array}{l}25,639,678 / \\
26730656\end{array}$ & PZE-104022764/PZE-104023674 & & & & & & 0.014 & 4.1 & C \\
\hline qRKP4-2 & 4 & $\begin{array}{l}41,851,034 / \\
56860597\end{array}$ & PZE-104033489/PZE-104041535 & & & -0.01 & 0.001 & 8.4 & & & $E 2, E 3, E 4, C$ \\
\hline qRKP4-3 & 4 & $\begin{array}{l}78,343,158 / \\
82013799\end{array}$ & PZE-104050391/PZE-104051877 & & & & & & 0.001 & 10.4 & $\mathrm{E} 2, \mathrm{E} 4, \mathrm{C}$ \\
\hline qRKP6 & 6 & $\begin{array}{l}141,080,410 / \\
161454721\end{array}$ & PZE-106083873/PZE-106115356 & -0.01 & 4.8 & & & & & & $\mathrm{E} 4, \mathrm{C}$ \\
\hline qRKP7-1 & 7 & $\begin{array}{l}39,125,424 / \\
46213540\end{array}$ & PZE-107030398/PZE-107033682 & -0.01 & 4.1 & & & & & & C \\
\hline$q R K P 7-2$ & 7 & $\begin{array}{l}140,421,815 / \\
141648137\end{array}$ & PZE-107084740/PZE-107086184 & -0.02 & 6.8 & & & & & & $\mathrm{E} 4, \mathrm{C}$ \\
\hline qRKP7-3 & 7 & $\begin{array}{l}143,113,852 / \\
143294688\end{array}$ & SYN35897/PZE-107088218 & & & & & & 0.003 & 3.5 & $C$ \\
\hline qRKP7-4 & 7 & $\begin{array}{l}149,763,210 / \\
149725744\end{array}$ & PZE-107094398/PZE-107094385 & & & -0.01 & 0.002 & 4.6 & & & $\mathrm{E} 4, \mathrm{C}$ \\
\hline qRKP10-1 & 10 & $\begin{array}{l}5,761,296 / \\
6537076\end{array}$ & PZE-110007326/PZE-110008811 & 0.01 & 5.4 & & & & & & C \\
\hline qRKP10-2 & 10 & $\begin{array}{l}131,104,195 / \\
134219127\end{array}$ & PZE-110074914/PZE-110079903 & & & 0.01 & 0.003 & 4.6 & -0.002 & 4.7 & $C$ \\
\hline $\begin{array}{l}\text { Total PVE } \\
(\%)\end{array}$ & & & & & 38.4 & & & 21.2 & & 42.7 & $C$ \\
\hline
\end{tabular}

${ }^{\mathrm{a}} \mathrm{Chr}$ Chromosome

${ }^{\mathrm{b}}$ The version 2 of the Maize B73 RefGen

${ }^{c} R I L$ the RILs, IF 2 the $I_{2}$ population, $M P H$ the MPH data set derived from the RILs and $\mathrm{IF}_{2}$ population

${ }^{\mathrm{d}} A$ additive effect, Negative additive values indicate that the allele for increasing trait value is contributed by the parent $08-641$, Positive additive values indicate

that the allele for increasing trait value is contributed by another parent YE478

ePVE phenotypic variance explained by QTL

${ }^{\mathrm{f}} D$ dominance effect

${ }^{9} E 1$ 2014Jinghong, E2 2015Jinghong, E3 2016Chongzhou, E4 2016Jinghong, C combined analysis across all environments

\section{Dominance and overdominance effects play crucial roles in heterosis for yield-related traits}

The contribution of dominance and overdominance to the heterosis phenomena was assessed by the $(|\mathrm{D} / \mathrm{A}|)$ ratio for each locus [34, 39] using single-marker analysis (SMA) and inclusive composite interval mapping (ICIM) (Fig. 4). Overdominance was detected for over $60 \%$ of genomic markers for EWPE and KWPE in both the $\mathrm{IF}_{2}$ and MPH/RIL datasets, more than $50 \%$ for KNPR and EL, over $40 \%$ for CWPE and ED, over $30 \%$ for CD and RKP, and over 15\% for RN. Based on SMA, the number of significant markers in the $\mathrm{IF}_{2}$ population showing overdominance accounted for $66.6 \%$ for KNPR, more than $40 \%$ for EWPE and KWPE, more than 15\% for EL, RKP, and ED, and less than $2 \%$ for CWPE, CD, and RN, respectively. Additionally, approximately $78 \%$ of QTLs for KWPE exhibited overdominance, as did $72.7 \%$ of QTLs for EWPE, 50\% of QTLs for KNPR, and less than $30 \%$ of QTLs for the remaining traits. Among traits, we also found a strong association between the proportion of QTLs with overdominance and the ratio of MPH/ mid-parent for $\mathrm{F}_{1}\left(r^{2}=0.951^{* * * *}\right)$ or the MPH level across hybrids from the $\mathrm{IF}_{2}$ population $\left(r^{2}=0.966^{* * * a}\right)$. We found, in general, significant but low correlations 


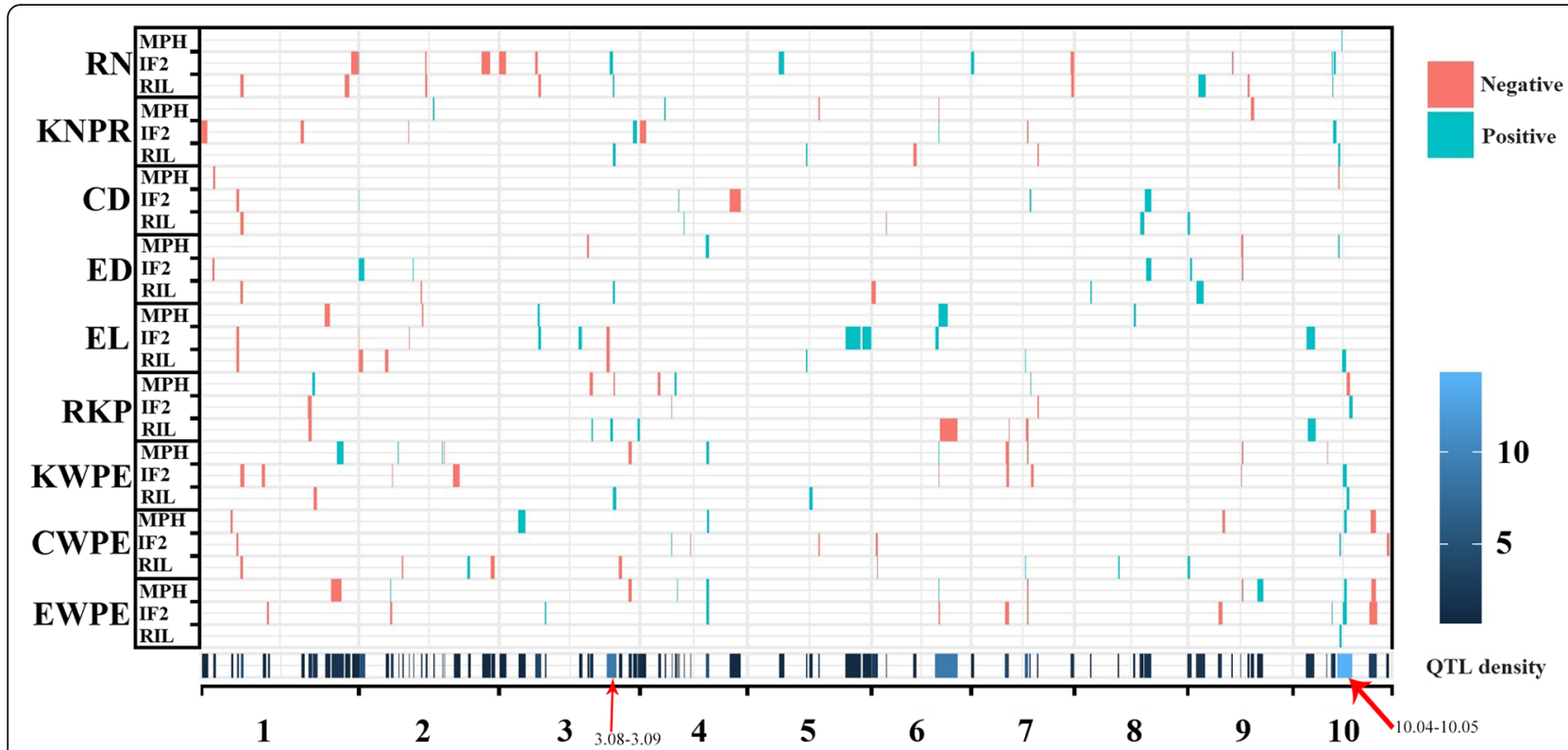

Fig. 3 Distribution of QTLs for yield-related traits detected in the RIL and IF populations and for heterosis. The red rectangles represent QTLS with negative effects (additive in the RIL population, dominant and additive in the $\mathrm{IF}_{2}$ population and dominance effects for mid-parent heterosis $(\mathrm{MPH})$ ), while the blue rectangles represent positive effects. The width of the rectangles indicates the confidence interval of QTLs. The heatmap of QTL density across ten chromosomes was indicated at the bottom of the figure and below the X-axis. EWPE, ear weight per ear; CWPE, cob weight per ear; EL, ear length; ED, ear diameter; CD, cob diameter; RN, row number; KNPR, kernel number per row; KWPE, kernel weight per ear; RKP, rate of kernel production

between performance per se or MPH of individuals in the $\mathrm{IF}_{2}$ population and their percentages of heterozygous marker loci (Additional file 8: Table S6).

\section{Analysis of EPI and QEI}

We estimated the total PVE by the epistatic loci in the whole-genome search using ICIM-EPI (Additional file 2: Figure S2). In the $\mathrm{IF}_{2}$ population, epistatic loci had higher PVE than single-locus QTLs for CWPE (50\% vs $41 \%$ ), ED (24\% vs $20 \%$ ), CD (56\% vs $32 \%$ ), and KNPR (53\% vs 37\%). In the MPH dataset, the total PVE by epistatic loci was greater than by single-locus QTLs for all traits except for EWPE (20\% vs $49 \%$ ), CWPE (23\% vs $28 \%$ ), KWPE ( $43 \%$ vs $52 \%$ ), and RKP ( $16 \%$ vs $43 \%$ ), indicating that EPI had a greater impact on these traits than single-locus QTLs, regardless of the statistical significance. The current findings also suggest that the cumulative effects resulting from combining the epistatic loci and single-locus QTLs explained a large proportion of PVE. Meanwhile, based on mixed-model-based composite interval mapping (MCIM) with the full QTL model, 28 pairs of marker intervals showed significant epistatic interaction effects; 13 additive by additive (AA) interactions were detected in the RIL population, 10 epistatic interactions in the $\mathrm{IF}_{2}$ population, and five digenic interactions in the MPH dataset (Additional file 9: Table S7). We observed 23 pairs of significant AA interactions, three for additive by dominance (AD) interactions, seven for dominance by additive (DA) interactions, and six for dominance by dominance (DD) interactions. Each marker interval interacted with one to five loci. In addition, a total of nine loci related to four traits exhibited significant additive $x$ environment interactions in the RIL and $\mathrm{IF}_{2}$ populations, seven in the RILs, and two in the $\mathrm{IF}_{2}$ population; only one locus, $q K W P E 2-2$, for the MPH level for KWPE showed a significant dominance $x$ environment interaction in the $\mathrm{IF}_{2}$ population (Additional file 10: Table S8). Many of these marker intervals involved in epistasis and QEI also contained QTLs with main effects on yield-related traits or MPH. For instance, the interval PZE-101184757/SYN25826 was involved in three significant AA interactions and contains $q R K P 1-1$.

\section{QTL co-localization across traits}

Overlapping QTLs for different traits were assigned to the same QTL cluster. In total, 95 QTLs were grouped into 33 QTL clusters distributed across all chromosomes (Table 3). Each QTL cluster contained QTLs for two to six traits. The cluster QC3-3 harboring six QTLs affected the means of EL, RN, ED, KNPR, RKP, and KWPE, as well as the MPH for RKP. QC10-3, within a $5 \mathrm{cM}$ region on chromosome 10 , had a pleiotropic effect on EWPE, KNPR, CWPE, and the MPH 


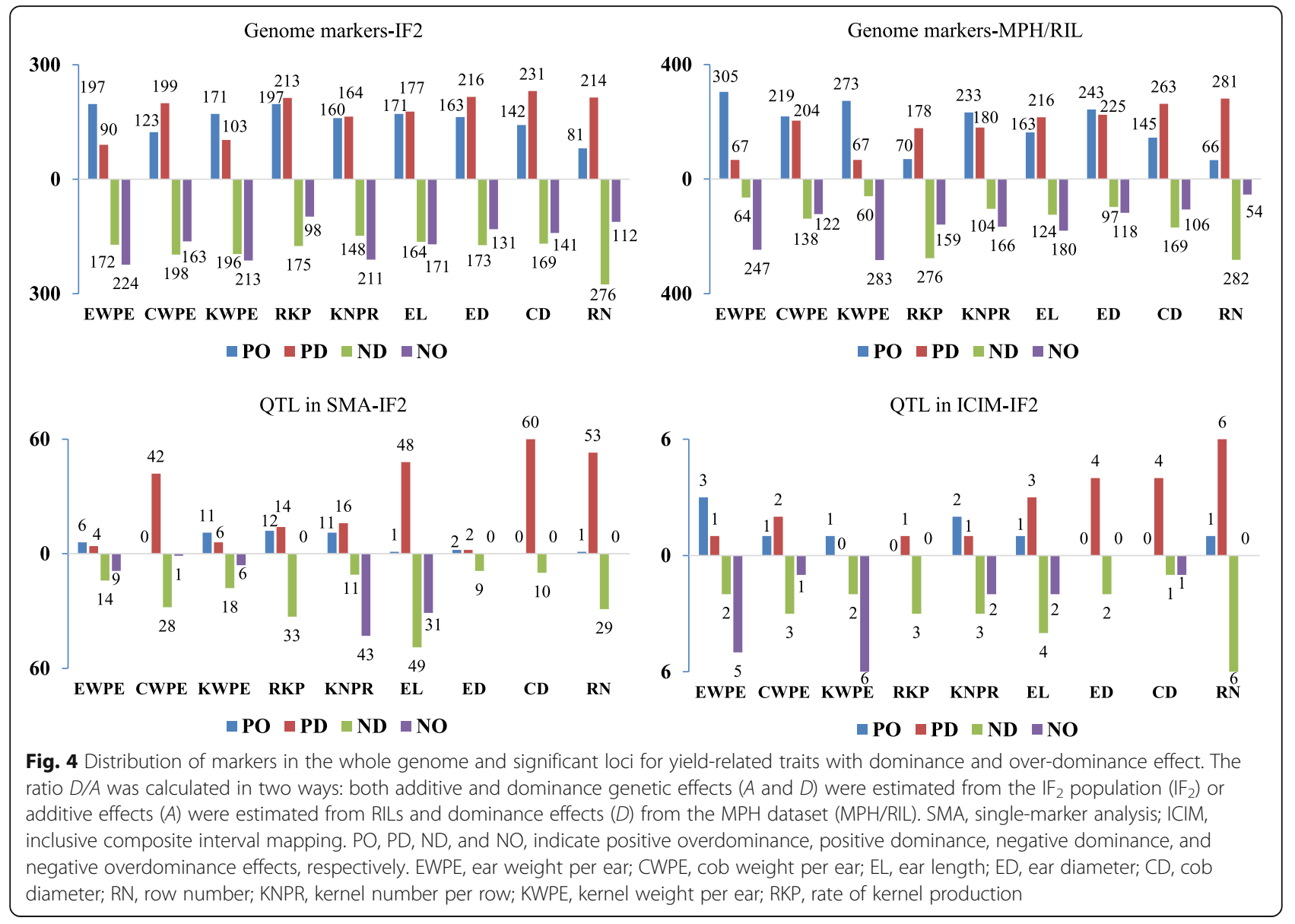

level of ED and CD. As expected from the moderate to high correlation between hybrid performance per se and $\mathrm{MPH}$, some loci associated with yield-related traits and MPH gathered together. Nearly $60 \%$ of QTL clusters were associated with two traits. Some of these clusters were specific for a determined population or for MPH. For example, clusters QC1-5 (bin 1.09-1.11) affected mid-parent heterosis for EWPE and KWPE. Contrarily, many other clusters comprised QTLs for yield-related traits and heterosis for those traits. Cluster QC1-4 (bin 1.08) affected KWPE and MPH for RKP. Genomic region 3.09 affected KNPR, RKP, and the MPH level of KWPE and EWPE. Interestingly, some of these clusters co-located in the interval of loci showing epistasis and QTL $\times$ environment interactions. For instance, QC7-1 (bin 7.02) had a pleiotropic effect on RKP, EWPE, KNPR, KWPE, and the MPH level for KWPE. This locus interacted with other loci exhibiting epistatic effects for RN in the RILs and for KWPE in the $\mathrm{IF}_{2}$ population. This hotspot also interacted with E1 (2014JH) for RKP in the RILs. These results further confirmed the complex genetic architecture of yield-related traits.

\section{Discussion}

Heterotic loci with dominance and epistasis and the complex genetic network of "crosstalk" contribute to grain yield

Previous studies $[29,31,34,40,41]$ proposed that better-parent heterosis was yield-related loci displaying dominance, overdominance, and/or epistasis. In the present study, over half of QTLs exhibited overdominance for KWPE, EWPE, and KNPR, which is similar to previous results reported by Frascaroli et al. [29] for overdominant loci for grain yield and kernel number per plant and by Guo et al. [34] for single markers and QTLs for grain yield. Such a high average degree of overdominance for grain yield and kernel number per plant could be, in part, explained by linkage in the repulsion of loci of the dominance type in mapping populations [29]. In accordance with this, greater number of genome markers with overdominance for EL and ED was previously observed in the $F_{2: 3}$ population, from which the current RIL population has been derived [42], than in the present mapping populations. The degree of dominance for the traits was well in accordance with the level of $F_{1}$ superiority over mid-parent performance and the 
Table 3 QTL clusters for yield-related traits and for heterosis for those traits detected in the RILand IF $F_{2}$ populations

\begin{tabular}{|c|c|c|c|c|c|c|}
\hline QTL Cluster $^{a}$ & $\operatorname{Bin}^{b}$ & Interval (cM) & Flanking marker & No. of QTL & Traits influenced $^{c}$ & References $^{\mathrm{d}}$ \\
\hline QC1-1 & 1.02 & $17.5-21.5$ & PZE-101026314/PZE-101027807 & 2 & ED (I), CD (M) & \\
\hline QC1-2 & 1.03 & $54.5-58.5$ & SYN25114/PZE-101058322 & 3 & EL $(I, R)$, CD (I), CWPE (I) & 67,35 \\
\hline QC1-3 & 1.03 & $60.5-66.5$ & SYN13385/SYN37775 & 5 & $\begin{array}{l}\text { ED (R), CD (R), CWPE R), } \\
\text { RN (R), KWPE (I) }\end{array}$ & 67,71 \\
\hline QC1-4 & 1.08 & $171.5-179.5$ & PZE-101187496/PZE-101196838 & 2 & RKP (M), KWPE (R) & 67,35 \\
\hline QC1-5 & $1.09-1.11$ & $200.5-219.5$ & PZE-101213558/SYN22772 & 2 & EWPE (M), KWPE (M) & $31,34,35$ \\
\hline QC2-1 & 2.02 & $0-8.5$ & PZE-102017304/SYN7604 & 4 & EL $(I, R), C D(I), E D(I), R N(I)$ & 35 \\
\hline QC2-2 & 2.04 & $48.5-52.5$ & PZE-102056295/PZE-102059924 & 2 & $\operatorname{EWPE}(M, I), \operatorname{KWPE}(M)$ & $29,35,71$ \\
\hline QC3-1 & 3.04 & $50.5-64.5$ & SYN28119/PZE-103036266 & 2 & $\mathrm{RN}(\mathrm{R}, \mathrm{I}), \mathrm{EL}(\mathrm{I}, \mathrm{M})$ & 67 \\
\hline QC3-2 & $3.06-3.07$ & $135.5-144.5$ & SYN23237/PZE-103136534 & 2 & ED (M), RKP (R, M) & \\
\hline QC3-3 & 3.08 & $165.5-180.5$ & SYN28063/PZE-103163529 & 6 & $\begin{array}{l}\text { EL }(I, R), \operatorname{RN}(I, R), \operatorname{RKP}(R, M) \\
\text { ED (R), KNPR (R), KWPE (R) }\end{array}$ & 35 \\
\hline QC3-4 & 3.09 & $189.5-217$ & PZE-103171163/PZE-103183391 & 4 & $\begin{array}{l}\text { KNPR (I), KWPE (M), EWPE (M), } \\
\text { RKP (R) }\end{array}$ & $34,37,71$ \\
\hline QC4-1 & 4.05 & $48.5-49.5$ & PZE-104033489/PZE-104041535 & 2 & CWPE (I), RKP (I) & \\
\hline QC4-2 & 4.06 & $101.5-106.5$ & PZE-104090796/PZE-104093153 & 4 & $\begin{array}{l}\text { EWPE (I, M), CWPE (M), ED (M), } \\
\operatorname{KWPE~(M)~}\end{array}$ & \\
\hline QC5-1 & 5.04 & $90.5-92.5$ & PZE-105093385/SYN32229 & 2 & EL (R), KNPR (R) & 67 \\
\hline QC5-2 & 5.04 & $109.5-111.5$ & PZE-105110168/PZE-105111323 & 2 & CWPE (I), KNPR (M) & 71 \\
\hline QC6-1 & $6.00-6.01$ & $0-9.5$ & $\begin{array}{l}\text { PUT-163a-94,473,612-4863/PZE- } \\
106008406\end{array}$ & 2 & ED (R), CWPE (R, I) & 71 \\
\hline QC6-2 & 6.05 & $98.5-105.5$ & PZE-106083557/PZE-106080884 & 4 & $\begin{array}{l}\text { EL }(I), \operatorname{EWPE}(I, M), \operatorname{KNPR}(I, M) \\
\operatorname{KWPE}(I, M)\end{array}$ & \\
\hline QC6-3 & $6.05-6.07$ & $103.5-132.5$ & PZE-106083873/PZE-106115356 & 3 & EL (M), RKP (R), KWPE (R) & 71 \\
\hline QC7-1 & 7.02 & $52.5-59.5$ & PZE-107019133/PZE-107033682 & 4 & $\begin{array}{l}\text { RKP (R), EWPE (I), KWPE }(I, M) \text {, } \\
\text { KNPR (R) }\end{array}$ & 35,71 \\
\hline QC7-2 & 7.03 & $83.5-84.5$ & PZE-107081442/PZE-107081254 & 2 & EL (R), CWPE (R) & 34 \\
\hline QC7-3 & 7.03 & $84.5-88.5$ & PZE-107084740/PZE-107086184 & 4 & $\begin{array}{l}\text { KNPR (I), EWPE (M, I), RKP (R), } \\
\text { KWPE (M) }\end{array}$ & 29 \\
\hline QC7-4 & 7.03 & $90.5-92.5$ & SYN35897/PZE-107088218 & 2 & CD (I), RKP (M) & \\
\hline QC7-5 & 7.03 & $102.5-104.5$ & PZE-107094398/PZE-107094385 & 2 & KNPR (R), RKP (I) & 35,71 \\
\hline QC8 & $8.05-8.06$ & $108.5-118.5$ & PZE-108074750/PZE-108096683 & 2 & CD (I), ED (I) & 71 \\
\hline QC9-1 & $9.00-9.01$ & $0-3.5$ & PZE-109000394/PZE-109001604 & 2 & CWPE (R), CD (R) & \\
\hline QC9-2 & $9.01-9.02$ & $13.5-27.5$ & PZE-109008839/PZE-109015923 & 2 & $\mathrm{ED}(\mathrm{R}), \mathrm{RN}(\mathrm{R})$ & 35,60 \\
\hline QC9-3 & 9.03 & $82.5-85.5$ & PZE-109037929//PZA03596.1 & 3 & $\begin{array}{l}\operatorname{KWPE}(I, M), \operatorname{EWPE}(M) \\
\operatorname{ED}(I, M)\end{array}$ & 71 \\
\hline QC10-1 & 10.02 & $20.5-34.5$ & PZE-110007326/PZE-110008811 & 2 & EL (I), RKP (R) & 29 \\
\hline QC10-2 & 10.03 & $59.5-66.5$ & SYN18227/PZE-110043216 & 3 & EWPE (I), KNPR (I), RN (R, I) & 60 \\
\hline QC10-3 & 10.04 & $69.5-74.5$ & PZE-110047164/PZE-110054264 & 5 & $\begin{array}{l}\text { ED (M), CD (M), EWPE (R), } \\
\text { KNPR (R), CWPE (I) }\end{array}$ & 29 \\
\hline QC10-4 & $10.04-10.05$ & $74.5-82.5$ & PZE-110054411/SYN17753 & 5 & $\begin{array}{l}\text { RN (M), EL (R), EWPE (I, M), } \\
\text { KWPE (I), CWPE (M) }\end{array}$ & 29,71 \\
\hline QC10-5 & 10.05 & $82.5-91.5$ & PZE-110074914/PZE-110079903 & 2 & $\operatorname{KWPE}(\mathrm{R}), \operatorname{RKP}(\mathrm{I}, \mathrm{M})$ & 35,71 \\
\hline QC10-6 & $10.06-10.07$ & $117.5-129.5$ & PZE-110095199/PZE-110104601 & 2 & EWPE $(I, M)$, CWPE (M) & 71 \\
\hline
\end{tabular}

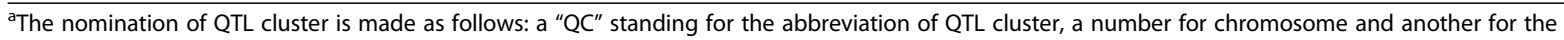
physical order

${ }^{\mathrm{b}}$ The genetic region included the position of QTL. (http://www.maizegdb.org/)

${ }^{\mathrm{C}} \mathrm{R}$, the RILs; I, the $\mathrm{IF}_{2}$ population; $\mathrm{M}$, mid-parent heterosis

d29, Frascaroli et al. 2007; 31, Tang et al. 2010; 67, Guo et al. 2011; 34, Guo et al. 2014; 35, Wang et al. 2016; 60, Liu et al. 2016; 37, Li et al. 2017; 71, Chen et al. 2017 
type of inheritance of different traits varied among those traits, which was consistent with other studies [29, 33, 34, 37, 43]. High correlation coefficients were observed between the proportion of QTLs displaying overdominance and the MPH levels across different traits. For example, the $F_{1}$ did not differ from the inbred parent lines $08-641$ and Ye478 for RN and CD, and accordingly QTLs, such as qRN1-2 and qRN7-2 displaying similar additive effects, detected for those traits showed low or no significant dominance effects and were mainly detected in RIL and $\mathrm{IF}_{2}$ populations [34]. The QTLs for EWPE, KNPR, and KWPE in $\mathrm{IF}_{2}$ population and the MPH dataset displayed high dominance effects. In general, similar dominance effects were found in the $\mathrm{IF}_{2}$ population and in the $\mathrm{MPH}$ dataset, but epistatic effects could interfere in the estimation of dominance effects and cause discrepancies between results obtained in different populations [44]. Discrepancies between additive effects estimated in the RIL and $\mathrm{IF}_{2}$ populations were even more conspicuous. For example, additive effects estimations for qCWPE6 were different when obtained from RIL or $\mathrm{IF}_{2}$ evaluations. These findings demonstrated that the loci displaying dominance contributed to yield-related traits and heterosis for those traits, although overdominance had an outstanding contribution to heterosis for EWPE, KWPE, and KNPR, which presented the highest values for $\mathrm{MPH}$.

The level of whole marker loci with heterozygosity and phenotypic variations (hybrid performance per se or MPH) were weakly correlated, whereas Frascaroli et al. [29] and Larièpe et al. [33] demonstrated moderate-tohigh correlations between them. Such low correlations indicated that the overall heterozygosity contributed little to hybrid performance per se or heterosis $[45,46]$. Huang et al. [46] demonstrated that phenotypic variations for yield-related traits are highly correlated with the number of accumulated superior gene alleles. In contrast, numerous epistatic effects and QTL $\times$ environment interactions for yield-related traits have been addressed in the literature [29, 31, 34, 37, 47-50]. The total PVE values by single-locus QTLs and epistasis together approach the high heritability for these yield-related traits [34], indicating the cumulative effects of different genetic effects [34, 41]. The QTL model, which was used to estimate the loci showing additive, dominance, and EPI effects, identified 28 pairs of significant loci with epistatic effects and 10 loci with QTL $\times$ environment interaction in the two mapping populations. Of those, only five pairs of epistatic loci were observed for $\mathrm{MPH}$. In addition, many of these loci showing interactions did not co-map with putative QTLs. Similar features were also reported by Yang et al. [51]. EPI and QEI are also involved in the complex genetic web for grain yield via "cross-talk" among loci $[45,47,48,52]$. Collectively, the cumulative effects of various genetic effects discussed above seem to explain the genetic mechanism of grain yield and betterparent performance [34, 41].

\section{QTL hotspots regulate the architecture of yield-related traits and heterosis}

Over $60 \%$ of QTLs gathered together and were integrated into 33 clusters distributed on 10 chromosomes. We found a good consistency for QTL clusters between our study and former studies (Table 3). Thirteen pleiotropic regions in the present study were consistently mapped to the clusters in all chromosomes, except in chromosome 4, that were defined by Chen et al. [53] in their meta-analysis of yield-related QTLs. Notably, many of these clusters related to MPH co-located with QTLs found in previous studies [29, 31, 34, 35] for better parent performance (Table 3). Cluster QC1-2 associated with EL, CD, and CWPE overlapped with the hotspot region for ear weight, grain weight, and KNPR between 38 and $42 \mathrm{Mb}$ on chromosome 1 , which was highlighted by Guo et al. [49], and with a genomic region containing QTLs for heterosis for grain yield, ear weight, kernel weight, and ear length reported by Wang et al. [35]. The heterosis locus QC1-5 (1.09-1.11) for EWPE and KWPE co-located with QTLs for heterosis for EWPE and KRP presented by Wang et al. [35]; with a heterosis locus for grain yield at approximately $271 \mathrm{Mbp}$ found by Tang et al. [31] using the heterotic pattern Mixed $\times$ Flint and by Larièpe et al. [33] using the pattern European flint $\times$ Dent; and with QTLs for 100-kernel weight and grain yield in an $\mathrm{IF}_{2}$ population reported by Guo et al. [34]. The genomic region 2.04 (QC2-2) controlling EWPE and the MPH level for EWPE and KWPE co-located with a QTL involved in heterosis for EL, RN, KWPE, KRP, EWPE, and grain yield by Wang et al. [35], and it was nearby the region implicated in heterosis for grain yield in the Reid $\times$ Lancaster pattern reported by Frascaroli et al. [29] and the meta-QTL for grain yield, ear-related traits, and kernel-related traits highlighted by Chen et al. [53]. Cluster QC3-3, being particularly interesting in the current study because of its association with heterosis for several traits, overlapped with a QTL hotspot for heterosis for EL, EWPE, RN, KRP, KWPE, and grain yield located between 209 and $213 \mathrm{Mbp}$ on chromosome 3 reported by Wang et al. [35]. Genomic region 3.09 (QC3-4), related to KNPR and RKP and the MPH level for KWPE and EWPE overlapped with the environmentally stable region for heterosis for KNPR, ear weight, and kernel weight between 218 and $228 \mathrm{Mbp}$ on chromosome 3 reported by $\mathrm{Li}$ et al. [37] in the heterotic pattern Reid $\times$ TSPT. Another hotspot region, QC10-3, related to five traits co-mapped with a heterotic locus that affects grain yield and kernel number presented 
by Frascaroli et al. [29]. In addition, genomic regions 2.04, 7.02, and 9.03, associated with heterosis for KWPE, contain QTLs for grain yield heterosis in the Reid $\times$ Lancaster heterotic pattern published by Schön et al. [32]. The cluster QC6-3 for RKP, KWPE, and the MPH level for EL overlapped with the heterotic loci for grain yield found by Samayoa et al. [36] in the population derived from an American dent $\times$ European flint cross. These results suggested the consistency of hotspot regions across different heterotic patterns, which may be used for genetic improvement of grain yield in the future.

In contrast, hybrid performance per se for yield-related traits and the MPH level for those traits were moderately to highly correlated and 13 QTLs found in the $\mathrm{IF}_{2}$ population co-localized with QTLs for MPH. These findings indicated the likely common genetic basis between grain yield and its MPH level. The results also partly supported that heterosis loci for grain yield are not independent from loci for grain yield, thus agreeing with Guo et al. [34] and refuting the conclusions by Tang et al. [31] who proposed independence between genomics involved in performance per se and heterosis.

\section{Genetic basis for grain yield and its components in different populations from the same parents}

Our study revealed that QTLs found in different populations derived from the same cross are not always consistent with previous studies [34, 54-56]. Among the 156 detected QTLs for yield-related traits and heterosis, only one QTL, $q R N 10-2$, was previously found across $\mathrm{F}_{2: 3}$ families derived from the cross $08-641 \times$ YE478 [60]. In addition, only nine QTLs with $|\mathrm{D} / \mathrm{A}|<1$ were simultaneously detected in the RILs and the $\mathrm{IF}_{2}$ population. Lack of congruency for QTLs found in populations derived from the same cross but with different homozygous levels could be due to the different genetic effects acting in each population, but also to the different environments used for evaluations and biases caused by population sampling [29].

\section{Conclusions}

Heterosis for yield-related traits in maize is one of the main issues for maize breeders, and investigation and assessment of heterotic loci involved in specific heterotic patterns are of particular interest. The inheritance of yield-related traits and MPH varied among different traits. A large proportion of the loci with dominance effect had a greater effect on most traits compared with loci with other genetic effects, whereas overdominance also contributed greatly to MPH for KNPR, EWPE, and KWPE in the current heterotic pattern. As QTL hotspots at 1.09-1.11, 2.04, 3.08-3.09, and 10.04-10.05 encompass genomic regions where other authors have found QTLs for heterosis across different heterotic patterns, markers in those regions could be used in marker-assisted selection programs for increased yield.

\section{Methods \\ Plant material and field experiments}

The mapping populations used in this study consisted of 301 RILs derived from a cross between the maize inbred lines 08-641 and Ye478. The elite inbred 08-641 comes from the Maize Research Institute of Sichuan Agricultural University, and Ye478 was provided by the Project of National Major Basic Dairy Research "973" Plan, which is developed by the Laizhou Academy of Agricultural Sciences [17]. The parental line 08-641 was crossed with Ye478 in the winter of 2010 to produce a set of RILs $\left(\mathrm{F}_{8}\right)$ by using the single seed descent method. The 301 RILs and the two parental lines were evaluated in four environments. Three environments were located at the Xishuangbanna maize breeding base of the Maize Research Institute of Sichuan Agricultural University, Jinghong $\left(\mathrm{JH} 21^{\circ} 95 \mathrm{~N}, 100^{\circ} 76 \mathrm{E}\right.$; average daytime temperature $18.6-21.9^{\circ} \mathrm{C}$; average rainfall per year 1200-1700 mm; tropical humid monsoon climate), in Yunnan province, in March 2014 (JH2014), March 2015 (JH2015), and March 2016 (JH2016), and one environment was located at the Modern Agriculture Research and Development Center of Sichuan Agricultural University, Chongzhou $\left(\mathrm{CZ} 30^{\circ} 33^{\prime} \mathrm{N}, 103^{\circ} 38^{\prime} \mathrm{E}\right.$; average daytime temperature $15.9^{\circ} \mathrm{C}$; average rainfall per year $1012.4 \mathrm{~mm}$; sub-tropical humid monsoon climate), in Sichuan province, April 2016 (CZ2016). An IF 2 population was developed with 320 RILs following the procedure of Hua et al. [57] and Tang et al. [31] The 320 RILs were randomly divided into two groups of 160 RILs. Then, the lines of both groups each RIL was only involved in a single cross, yielding $160 \mathrm{~F}_{1}$ crosses. We repeated the procedure mentioned above twice and constructed 320 crosses that constituted the $\mathrm{IF}_{2}$ population. After genotyping the RILs, those with heterozygosity above $20 \%$ and their respective crosses were removed. After filtering the RILs, the $\mathrm{IF}_{2}$ population consisted of 298 crosses that were evaluated under three environments $(2015 \mathrm{JH}, 2016 \mathrm{JH}$, and 2016CZ). In each environment, each trial was performed following a randomized complete block design with two repetitions. Plant density was 57,000 plants per ha. Each plot consisted of a single row. Each row with 14 plants was $3 \mathrm{~m}$ long, with $0.80 \mathrm{~m}$ space between rows. The trials with two mapping populations were adjacent and occupied a uniform and square parcel. Field management was in accordance with local practices.

\section{Phenotypic measurements and analysis}

In each plot, 10 well-pollinated ears were randomly chosen from all ears harvested. The nine yield-related traits measured (Additional file 3: Table S1) were: EL 
(cm), ED (mm), CD (mm), RN (count), KNPR, EWPE (g), CWPE (g), KWPE (g), and RKP. Means for each replication were used for further analyses. The distribution of the phenotypic traits and the Pearson's correlation, using PROC CORR from the statistical software package SPSS 17.0 (SPSS, Inc., Chicago, IL, USA), were conducted with the means of the phenotypic traits in the 301 RILs and 298 hybrids of the $\mathrm{IF}_{2}$ population across all environments. A hierarchical cluster analysis ("hclust") was carried out for phenotypic traits, based on the standardized data from the RILs [58, 59]. Combined analyses of variance of the RILs and the $\mathrm{IF}_{2}$ population were computed for each trait using the GLM procedure in SPSS 17.0 (SPSS, Inc., Chicago, IL, USA) with genotype as a fixed effect and replication and environment as random effects. Broad-sense heritability $\left(h^{2}\right)$ for the RILs and $\mathrm{IF}_{2}$ population was computed on an entry mean basis as described by Hallauer and Miranda [60]:

$$
h^{2}=\sigma_{g}^{2} /\left(\sigma_{g}^{2}+\frac{\sigma_{g e}^{2}}{n}+\frac{\sigma^{2}}{n b}\right),
$$

where $\sigma_{g}^{2}$ represents the genetic variance, $\sigma_{g e}^{2}$ represents the genotype $\times$ environment interaction variance, $\sigma^{2}$ represents the error variance, $b$ represents the number of replications, and $n$ is the number of environments. The $90 \%$ confidence interval of heritability $\left(h^{2}\right)$ was determined according to Knapp et al. [61]. Each phenotypic trait of the 301 RILs and each trait of the $\mathrm{IF}_{2}$ population were analyzed following two mixed models fitted by restricted maximum likelihood:

$$
\begin{aligned}
& Y_{m k}=\mu+G_{m}+R_{k}+\varepsilon_{m k}, \\
& Y_{\text {mik }}=\mu+G_{m}+G E_{m i}+E_{i}+R_{k}+\varepsilon_{m i k},
\end{aligned}
$$

where $Y_{m k}$ is the phenotypic value of genotype $\mathrm{m}$ in replication k; $Y_{m i k}$ indicates the phenotypic value of genotype $m$ in environment $i$ and replication $k$; $\mu$ is the overall mean of the RIL population or the $\mathrm{IF}_{2}$ population; $G_{m}$ is the random effect of genotype $m ; G E_{m i}$ is the random effect of the interaction between genotype $m$ and environment $i ; E_{i}$ is the random effect of environment $i ; R_{k}$ is the random effect of replication $k$; and $\varepsilon_{m k}$ and $\varepsilon_{\text {mik }}$ denote the random experimental error. The best linear unbiased predictor values (BLUPs) with Eqs. (2) and (3) were used for single and combined environment analysis, respectively. The adjusted mean BLUP values of each RIL across four environments and each hybrid across three environments were used for QTL mapping. The analyses were conducted with the $R$ program for statistical computing $[59,62]$. Besides, MPH was estimated as [40]:

$$
\mathrm{MPH}_{12}=\mathrm{F}_{12}-\left(\mathrm{P}_{1}+\mathrm{P}_{2}\right) / 2
$$

where $F_{12}$ is the genotypic value of the $F_{12}$ individual from the $\mathrm{IF}_{2}$ population, and $\left(\mathrm{P}_{1}+\mathrm{P}_{2}\right) / 2$ is the average of BLUP values of the corresponding parents $\left(P_{1}\right.$ and $P_{2}$ from the RIL population) estimated from the RIL evaluation. The genotypic dataset for MPH depends on dominance and epistatic effects, but epistatic effects were not included and an additive-dominance model was assumed for the $\mathrm{IF}_{2}$ population [31, 34].

\section{Molecular linkage map construction}

Following the modified CTAB protocol [63], DNA was isolated from 7-day-old seedling leaves of the 301 RILs and the parents grown in shade (10 plants per RIL as a bulk). The oligonucleotide pool assay consisted of 3072 well-distributed, high-quality single nucleotide polymorphisms (SNPs) from all 10 maize chromosomes that were selected from 56,110 SNPs in 513 maize inbred lines developed by the National Maize Improvement Center of China using Illumina GoldenGate technology. The protocol for genotyping SNPs using an Illumina BeadStation 500 G (Illumina, San Diego, CA, USA) was conducted as described by Fan et al. [64]. Marker data from the RILs were filtered for heterozygous data points $(<20 \%)$, missing data points $(<20 \%)$, and segregation distortion (in accordance with the expected Mendelian segregation ratio of 1:1). A total of 683 SNPs selected for their uniform distribution throughout all 10 maize chromosomes were used to construct the linkage map with a total genetic length of $1786.1 \mathrm{cM}$ and an average interval distance of $2.61 \mathrm{cM}$. The genotypes of each cross from the $\mathrm{IF}_{2}$ population were deduced from the marker genotypes of their RIL parents, with QTL mapping for the $\mathrm{IF}_{2}$ population and the $\mathrm{MPH}$ dataset performed using the molecular linkage map of the RIL population [31, 57]. The genetic map for the RILs and $\mathrm{IF}_{2}$ population was developed using MapDisto 1.7.5 [65]. The Kosambi mapping function was used for converting recombination frequencies to genetic distances [66]. The average proportion of homozygous loci for YE478 variants, homozygous loci for 08-641 variants, and heterozygous loci in each individual of the $\mathrm{IF}_{2}$ population was $25.2,24.7$, and $50.1 \%$, respectively, according to the expected ratio in an $\mathrm{F}_{2}$ population.

\section{QTL mapping}

QTL mapping for each trait in the RIL and $\mathrm{IF}_{2}$ populations and for MPH using both populations was performed using QTL ICIMapping software [67] with ICIM $[68,69]$. For QTL detection on ICIM-ADD, the largest $p$-value for entering variables in stepwise regression of residuals on marker variables, PIN was set to 0.001 . The largest $p$-value for removing variables is assumed to be two times the PIN. The threshold logarithm of the odds (LOD) score to declare significant QTL was calculated using the 1000 permutation test at Type I error of 0.05 with the step size of 
$1 \mathrm{cM}$ was 2.5 for the RILs, $\mathrm{IF}_{2}$ population, and the $\mathrm{MPH}$ dataset since very minor differences were observed in LOD score among them. Under the additive-dominance model assumed, only QTLs with dominant effect were detected for MPH [34]. We also performed single marker analysis (SMA) to detect QTLs with the same empirical threshold LOD mentioned previously, and the degree of dominance was calculated as a ratio of dominance to additive effects $(|\mathrm{D} / \mathrm{A}|)$ for each QTL [34]. Loci with $|\mathrm{D} / \mathrm{A}|$ values greater than 1.26 displayed overdominance; otherwise, the QTL was treated as dominance type [39]. The QTLs were nominated as follows: a lowercase letter "q" standing for QTL, the trait abbreviation, one number standing for the chromosome and another for the physical order or the QTL within the chromosome. The QTLs for different traits detected in overlapping or adjacent intervals were regarded as a QTL cluster. The graphical presentation of linkage maps and QTLs were generated with the $R$ program [59]. To scan for significant marker interval interactions, epistasis interaction (EPI, digenic interactions; $\mathrm{AA}, \mathrm{AD}, \mathrm{DA}$, and $\mathrm{DD}$ ) was identified with the step in scanning of $5 \mathrm{cM}$ and PIN $=0.0001$ on ICIM-EPI. An empirical threshold LOD for digenic epistasis interaction was set at 5. Meanwhile, the QTL Network program 2.1 based on MCIM [70-72] was also used to identify QTL with AA effects in the RILs; AA, AD, DA, and DD effects in the $\mathrm{IF}_{2}$ population using combined data across all environments and the MPH dataset using the average data across environments. QTL $\times$ environment interaction effects $(\mathrm{QEI})$ in the RIL and $\mathrm{IF}_{2}$ population were estimated for yield-related traits via combined analysis across all environments. The testing window and filtration window size was set at $10 \mathrm{cM}$ with a walk speed set to $2 \mathrm{cM}$ for one-dimensional genome scans. The F-statistic and the critical F-value were estimated with the help of 1000-permutations test [73] with $p<0.05$ as experiment-wise significance level for candidate interval selection. The final full QTL model for each trait incorporated significant additive, dominance, and epistatic effects, as well as their interactions with environments. Bonferroni correction was used to compute the comparison-wise significance threshold assuming an experiment-wise error $<0.05$ [74]. We also calculated the percentage of heterozygous loci for each RIL analyzed the correlation relationships between phenotypic performance and heterozygosity, and conducted regression analyses of phenotypic performance on heterozygosity [59].

\section{Additional files}

Additional file 1: Figure S1. The performance of parental inbreds of the RIL population (YE478 and 08-641) and the hybrid F1 across four environments. The Duncan multiple range test was used for the comparison of means. Genotypes with lower-case letters were significantly different at the 0.05 probability level. Means with same letters are not significantly different. The data are shown as means \pm SD (standard deviation). EWPE, ear weight per ear; CWPE, cob weight per ear; $E L$, ear length; ED, ear diameter; CD, cob diameter; RN, row number; KNPR, kernel number per ear; KWPE, kernel weight per ear; RKP, rate of kernel production. (JPG $513 \mathrm{~kb}$ )

Additional file 2: Figure S2. Phenotypic variance explained (PVE) by QTL and epistasis interaction (EPI, digenic interaction) effects for nine yield-related traits in the RILs, IF 2 population and the MPH dataset. The total PVE for QTL and epistasis was estimated on ICIM-ADD and ICIM-EPI, respectively. ICIM, inclusive composite interval mapping. RIL, recombinant inbred line. $\mathrm{IF}_{2}$, immortalized $\mathrm{F}_{2}$. MPH, mid-parent heterosis. EWPE, ear weight per ear; CWPE, cob weight per ear; EL, ear length; ED, ear diameter; $\mathrm{CD}$, cob diameter; RN, row number; KNPR, kernel number per ear; KWPE, kernel weight per ear; RKP, rate of kernel production. (PPTX $37 \mathrm{~kb}$ )

Additional file 3: Table S1. List of abbreviations and definitions for the yield-related traits recorded at harvest in a sample of ten ears per plot. (DOCX $16 \mathrm{~kb}$ )

Additional file 4: Table S2. The performance of yield-related traits in the RILs, the $\mathrm{IF}_{2}$ population and the MPH dataset across all environments. EWPE, ear weight per ear; CWPE, cob weight per ear; ED, ear diameter; $C D$, cob diameter; $E L$, ear length; RN, row number; KNPR, kernel number per row; KWPE, kernel weight per row; RKP, rate of kernel production. ${ }^{a}$ F1, The cross of 08-641 $\times$ YE478. RIL, the recombinant inbred lines. ${ }^{b} I_{2}$ and $\mathrm{MPH}$ indicate the immortalized $\mathrm{F}_{2}$ population and mid-parent heterosis, respectively. ${ }^{C}$ Mean \pm SE represents mean across all environments \pm standard error. ${ }^{d} \mathrm{MPH}(\%)=\left(\mathrm{F}_{1}-\left(\mathrm{P}_{1}+\mathrm{P}_{2}\right) / 2\right) /\left(\left(\mathrm{P}_{1}+\mathrm{P}_{2}\right) / 2\right)^{*} 100 \%$. (DOCX $\left.18 \mathrm{~kb}\right)$

Additional file 5: Table S3. Pearson's correlation coefficients among yield-related traits in the RILs ${ }^{a}$ across four environments, the $\mathrm{IF}_{2}{ }^{\mathrm{c}}$ populations across three environments, and the mid-parent heterosis (MPH) dataset. * Significant at the 0.05 probability level. ** Significant at the 0.01 probability level. The absolute values of Pearson's correlation coefficients $\left(r^{2}\right)$ with bold are equal to or larger than 0.5. EWPE, ear weight per ear; CWPE, cob weight per ear; ED, ear diameter; $C D$, cob diameter; $E L$, ear length; $R N$, row number; KNPR, kernel number per row; KWPE, kernel weight per row; RKP, rate of kernel production. ${ }^{a}$ Pearson's correlation coefficients $\left(r^{2}\right)$ in the first row for each trait are for the RILs. ${ }^{b}$ Pearson's correlation coefficients $\left(r^{2}\right)$ in the second row for each trait are for the $\mathrm{IF}_{2}$ population. ${ }^{\mathrm{C}}$ Pearson's correlation coefficients $\left(r^{2}\right)$ in the third row for each trait are for mid-parent heterosis. (DOCX $1433 \mathrm{~kb})$

Additional file 6: Table S4. Main feature of QTLs for yield-related traits and for mid-parental heterosis for those traits from the RILs and $\mathrm{F}_{2}$ population via single environment analysis. ${ }^{a}$ The nomination of QTL is made as follows: a " $q$ " standing for the abbreviation of QTL, the abbreviation of the trait, an "S" standing for single environment analyses, a number standing for chromosome, and another for physical order. EWPE, ear weight per ear; CWPE, cob weight per ear; ED, ear diameter; $C D$, cob diameter; EL, ear length; $R N$, row number; KNPR, kernel number per row; KWPE, kernel weight per row; RKP, rate of kernel production. ${ }^{b} \mathrm{RIL}$, recombinant inbred lines; $\mathrm{F}_{2}$, the immortalized $\mathrm{F}_{2} ; \mathrm{MPH}$, mid-parental heterosis derived from the RIL and $\mathrm{IF}_{2}$ populations. ' Env, environment; E1, 2014Jinghong; E2, 2015Jinghong; E3, 2016Chongzhou; E4, 2016Jinghong. ' Chr, chromosome. ${ }^{e}$ A, additive effect; Negative additive values indicate that the allele for increasing trait value is contributed by the parent 08-641; Positive additive values indicate that the allele for increasing trait value is contributed by another parent $Y e 478 .{ }^{f} \mathrm{D}$, dominance effect. ${ }^{9}$ PVE, phenotypic variance explained by QTL. (DOCX $70 \mathrm{~kb}$ )

Additional file 7: Table S5. Pearson's correlation coefficients between phenotypic performance per se in $\mathrm{IF}_{2}$ population and mid-parent heterosis for those traits. ${ }^{* * *}$ Significant at $p<0.0001$. (DOCX $15 \mathrm{~kb}$ )

Additional file 8: Table S6. Pearson's correlation coefficients between phenotypic variation and genome marker heterozygosity. ${ }^{a}$ Correlation between performance per se in $\mathrm{IF}_{2}$ population and percentage of heterozygous loci. ${ }^{b}$ Correlation between mid-parent heterosis and the percentage of heterozygous loci. ${ }^{* * *}$, significant at $p<0.0001$ level; ${ }^{* *}$, significant at $p<0.01$ level; , significant at $p<0.05$ level; NS, not significant. EWPE, ear weight per ear; CWPE, cob weight per ear; ED, ear diameter; CD, cob diameter; EL, ear length; RN, row number; KNPR, kernel number per row; KWPE, kernel weight per row; RKP, rate of kernel production. (DOCX 16 kb) 
Additional file 9: Table S7. Epistatic interactions for yield-related traits and mid-parent heterosis detected in the RILs and $\mathrm{IF}_{2}$ population across all environments. ${ }^{2}$ EWPE, ear weight per ear; CWPE, cob weight per ear; $C D$, cob diameter; EL, ear length; RN, row number; KNPR, kernel number per row; KWPE, kernel weight per row; RKP, rate of kernel production. ${ }^{b}$ Interval of the first QTL i. ${ }^{C}$ Chromosome ID at the first scanning position. ${ }^{\mathrm{d}} \mathrm{A}$ Additive effect of the QTL i. ${ }^{e}$ D i Dominance effect of the QTL i. ${ }^{f}$ Interval of the second QTL j. ${ }^{g}$ Chromosome ID at the second scanning position. ${ }^{\mathrm{h}} \mathrm{A} \_$j Additive effect of the QTL j. ${ }^{i}$ D_j Dominance effect of the QTL j. ${ }^{j}$ AA: Estimated additive by the additive effect of QTLs at the two scanning positions. ${ }^{k}$ AD: Estimated additive by dominance effect of QTLs at the two scanning positions. ${ }^{m}$ DA: Estimated dominance by the additive effect of QTLs at the two scanning positions. ${ }^{n} \mathrm{DD}$ : Estimated dominance by dominance effect of QTLs at the two scanning positions. ${ }^{2}(\%)$ : Heritability for epistatic QTL effects. The interval in bold was co-located in QTLs associated with the same trait via combined analysis across all environments. , , ${ }^{* * * *+}$ indicate significance at $p<0.05, p<0.01$, and $p<0.0001$, respectively. (DOCX $26 \mathrm{~kb}$ )

Additional file 10: Table S8. QTL $\times$ environment interactions for yieldrelated traits detected in the RILs under four environments and the $\mathrm{IF}_{2}$ population under three environments. ${ }^{a}$ RIL, recombinant inbred lines; $I_{2}$ the immortalized $\mathrm{F}_{2} \cdot{ }^{\mathrm{b}} \mathrm{AE}$ indicates the additive by designed environment interaction effect. E1, E2, E3, and E4, represent 2014JH, $2015 \mathrm{JH}, 2016 \mathrm{CZ}$, and $2016 \mathrm{JH}$, respectively. ${ }^{c} h^{2}(\mathrm{AE}, \%)$ is the contribution rate of additive by environment interaction effect. ${ }^{d}$ DE indicates the dominance by

designed environment interaction effect. E1, E2, E3, and E4, represent 2014JH, 2015JH, 2016CZ, and 2016JH, respectively. ${ }^{e} h^{2}(\mathrm{DE}, \%)$ is the contribution rate of dominance by environment interaction effect. ${ }^{*},{ }^{* * *}$, ${ }^{* * *}$ indicate significance at $p<0.05, p<0.01$, and $p<0.0001$, respectively. The interval in bold was co-located in QTLs associated with the same trait via combined analysis across all environments. (DOCX $21 \mathrm{~kb}$ )

Additional file 11: Genotypic and phenotypic data of the RIL and IF populations for the QTL analyses. (XLSX $1583 \mathrm{~kb}$ )

\section{Abbreviations}

AA: Additive $\times$ additive interaction; AD: Additive $\times$ dominance interaction; BLUP: Best linear unbiased predictor; CD: Cob diameter; CWPE: Cob weight per ear; DA: Dominance $\times$ additive interaction; DD: Dominance $\times$ dominance interaction; ED: Ear diameter; EL: Ear length; EPI: Epistatic interaction; EWPE: Ear weight per ear; ICIM: Inclusive composite interval mapping; $\mathrm{IF}_{2}$ : The immortalized $\mathrm{F}_{2} ; \mathrm{KNPR}$ : Kernel number per row; KWPE: Kernel weight per ear; MCIM: Mixed-model-based composite interval mapping; MPH: Midparent heterosis; QTL: Quantitative trait loci; RIL: Recombinant inbred lines; RKP: Rate of kernel production; RN: Row number; SMA: Single marker analysis; SNP: Single nucleotide polymorphism

\section{Acknowledgements}

We thank Dr. Ana Butrón and Dr. Pedro Revilla of Misión Biológica de Galicia (CSIC) for valuable suggestions and careful corrections, and also thank the help of Dr. Zhengjie Chen of Maize Research Institute for the construction of the $\mathrm{IF}_{2}$ population, and all students for participating in the fieldwork.

\section{Authors' contributions}

YBH, YHL, and XBH designed the study. YHL and XBH developed the RILS, and $\mathrm{QY}$ and $\mathrm{HL}$ constructed the $\mathrm{IF}_{2}$ population. All coauthors conducted the experiments of phenotypic identification in the field. YHL, XBH, and QY analyzed the data. YHL and YQ prepared the manuscript, and all authors read and approved the final manuscript.

\section{Funding}

The current research was supported by the Project of National Major Basic Dairy Research "973" Plan (\#2014CB138202 and \#2011CB100106) and Science and Technology Plan Projects in Sichuan Province (\#2016JY0065). The funders had no role in the design of the study and collection, analysis, and interpretation of data and in writing the manuscript.

\section{Availability of data and materials}

Supporting data are available in Additional file 11, and materials are available from the authors upon request.
Ethics approval and consent to participate

Not applicable.

\section{Consent for publication}

Not applicable.

\section{Competing interests}

The authors declare that they have no competing interests

\section{Author details}

${ }^{1}$ State Key Laboratory of Crop Gene Exploration and Utilization in Southwest China, Sichuan Agricultural University, Chengdu 611130, China. ${ }^{2}$ College of Agronomy, Sichuan Agricultural University, Chengdu 611130, China. ${ }^{3}$ Maize Research Institute, Sichuan Agricultural University, Chengdu 611130, China.

${ }^{4}$ College of Agriculture and Food Engineering, Baise University, Baise 533000, Guangxi, China. ${ }^{5}$ College of Life Science, Sichuan Agricultural University, Ya'an 625014, China.

Received: 3 April 2019 Accepted: 30 August 2019

Published online: 09 September 2019

\section{References}

1. Duvick DN. Heterosis: feeding people and protecting natural resources. In: Coors JG, Pandey S, editors. Proceedings of the International Symposium on the Genetics and Exploitation of Heterosis in Crops, CIMMYT, Mexico City, 17-22 Aug 1997. Madison: ASA, CSSA, and SSSA; 1999. p. 19-29.

2. Duvick DN. Long-term selection in a commercial hybrid maize breeding program. In: Janick J, editor. Plant breeding reviews 24, part 2. Hoboken: Wiley; 2004. p. 109-51.

3. Duvick DN. The contribution of breeding to yield advances in maize. Adv Agron. 2005;86:83-145.

4. Ci X, Li M, Liang X, Xie Z, Zhang D, Li X, et al. Genetic contribution to advanced yield for maize hybrids released from 1970 to 2000 in China. Crop Sci. 2011;51:13-20

5. Moll RH, Lonnquist JH, Velez FJ, Johnson C. The relationship of heterosis and genetic divergence in maize. Genetics. 1965;52:139-44.

6. Moll RH, Salhuana WS, Robinson HF. Heterosis and genetic diversity in variety crosses of maize. Crop Sci. 1962;2:197-8.

7. Smith OS, Smith JS, Bowen SL, Tenborg RA, Wall SJ. Similarities among a group of elite maize inbreds as measured by pedigree, F1grain yield, grain yield, heterosis, and RFLPs. Theor Appl Genet. 1990;80:833-40.

8. Beck DL, Vasal SK, Crossa J. Heterosis and combining abilityamong subtropical and temperate intermediate-maturity maize germplasm. Crop Sci. 1991;31:68-73.

9. Messmer MM, Melchinger AE, Boppenmaier J, Herrmann RG, Brunklaus-Jung E. RFLP analysis of early maturing European maize germplasm. I. Genetic diversity among flint and dent inbreds. Theor Appl Genet. 1992;83:1003-12.

10. Messmer MM, Melchinger AE, Herrmann R, Boppenmaier J. Relationship among early maturing European maize hybrids. II Crop Sci. 1993;33:944-50.

11. Troyer AF. Background of U.S. hybrid corn. Crop Sci. 1999;39:601-26.

12. Troyer AF. Background of U.S. hybrid corn. II. Crop Sci. 2004;44:370-80.

13. Teng WT, Cao JS, Chen YH, Liu XH, Jing XQ, Zhang FJ, et al. Analysis of maize heterotic groups and patterns during past decade in China. Sci Agric Sin. 2004;37:1804-11.

14. Lu H, Bernardo R. Molecular marker diversity among current and historical maize inbreeds. Theor Appl Genet. 2001;103:613-7.

15. Smith CW, Betrán J, Runge E. Corn: origin, history, technology, and production. Hoboken: Wiley; 2004.

16. Mikel MA, Dudley JW. Evolution of north American dent corn from public to proprietary germplasm. Crop Sci. 2006;46:1193-205.

17. Li Y, Wang TY. Germplasm base of maize breeding in China and formation of foundation parents. J Maize Sci. 2010;18:1-8.

18. Zhang S, Li X, Yuan L, Li M, Peng Z. Heterotic groups and exploitation of heterosis-methodology, strategy, and use in hybrid maize breeding in China. In: Srinivasan G, Zaidi PH, Prasanna BM, Gonzalez F, Lesnick K, editors. Proceedings of the Eight Asian Regional Maize Workshop: New technologies for the new millennium. Bangkok; 2002. p. 64-9.

19. Shull GH. The composition of a field of maize. American Breeders Assoc Rep. 1908;: 296-301.

20. Shull GH. What is heterosis? Genetics. 1948:33:439-46.

21. Davenport CB. Degeneration, albinism and inbreeding. Science. 1908;28:454-5. 
22. Bruce $A B$. The Mendelian theory of heredity and the augmentation of vigor. Science. 1910;32:627-8.

23. East EM. Inbreeding in corn. Rep Conn Agric Exp Stn. 1907;1908:419-28.

24. Powers L. An expansion of Jones's theory for the explanation of heterosis. Am Nat. 1944;78:275-80.

25. Williams W. Heterosis and the genetics of complex characters. Nature. 1959; 184:527-30.

26. Stuber CW, Lincoln SE, Wolff DW, Helentjaris T, Lander ES. Identification of genetic factors contributing to heterosis in a hybrid from two elite maize inbred lines using molecular markers. Genetics. 1992;132:823-39.

27. Cockerham CC, Zeng ZB. Design III with marker loci. Genetics. 1996;143:1437-56.

28. Lu H, Romero-Severson J, Bernardo R. Genetic basis of heterosis explored by simple sequence repeat markers in a random-mated maize population. Theor Appl Genet. 2003;107:494-502.

29. Frascaroli E, MA C', Landi P, Pea G, Gianfranceschi L, Villa M, et al. Classical genetic and quantitative trait loci analyses of heterosis in a maize hybrid between two elite inbred lines. Genetics. 2007;176:625-44.

30. Garcia AAF, Wang SC, Melchinger AE, Zeng ZB. Quantitative trait loci mapping and the genetic basis of heterosis in maize and rice. Genetics. 2008;180:1707-24.

31. Tang JH, Yan JB, Ma XQ, Teng WT, Wu WR, Dai JR, et al. Dissection of the genetic basis of heterosis in an elite maize hybrid by QTL mapping in an immortalized $F_{2}$ population. Theor Appl Genet. 2010;120:333-40.

32. Schön CC, Dhillon BS, Utz HF, Melchinger AE. High congruency of QTL positions for heterosis of grain yield in three crosses of maize. Send to Theor Appl Genet. 2010;120(2):321-32.

33. Larièpe $A$, Mangin $B$, Jasson $S$, Combes V, Dumas $F$, Jamin $P$, et al. The genetic basis of heterosis: multiparental quantitative trait loci mapping reveals contrasted levels of apparent overdominance among traits of agronomical interest in maize (Zea mays L.). Genetics. 2012;190:795-835.

34. Guo T, Yang N, Tong H, Pan Q, Yang X, Tang J, et al. Genetic basis of grain yield heterosis in an "immortalized $F_{2}$ " maize population. Theor Appl Genet. 2014;127:2149.

35. Wang H, Zhang X, Yang H, Liu X, Li H, Yuan L, et al. Identification of heterotic loci associated with grain yield and its components using two CSSL test populations in maize. Sci Rep. 2016;6:38205.

36. Samayoa LF, Malvar RA, Butrón A. QTL for maize Midparent Heterosis in the heterotic pattern American dent $\times$ European Flint under corn borer pressure. Front Plant Sci. 2017;8:573.

37. Li H, Yang Q, Gao L, Zhang M, Ni Z, Zhang Y. Identification of heterosisassociated stable QTLs for ear-weight-related traits in an elite maize hybrid Zhengdan 958 by design III. Front Plant Sci. 2017:8:561.

38. Wang $Y$, Zhang $X$, Shi $X$, Sun $C$, Jin J, Tian R, et al. Heterotic loci identified for maize kernel traits in two chromosome segment substitution line test populations. Sci Rep. 2018;8:11101.

39. Falconer DS, Mackay TFC. Introduction to quantitative genetics. 4th ed. Essex: Longman; 1996.

40. Hua JP, Xing YZ, Wei WR, Xu CG, Sun XL, Yu SB, et al. Single-locus heterotic effects and dominance by dominance interactions can adequately explain the genetic basis of heterosis in an elite rice hybrid. Proc Natl Acad Sci U S A. 2003;100:2574-9.

41. Zhou G, Chen Y, Yao W, Zhang C, Xie W, Hua J, et al. Genetic composition of yield heterosis in an elite rice hybrid. Proc Natl Acad Sci U S A. 2012;109:15847-52.

42. Liu Y, Hou X, Xiao Q, Yi Q, Bian S, Hu Y, et al. Genetic analysis in maize foundation parents with mapping population and testcross population: Ye478 carried more favorable alleles and using QTL information could improve foundation parents. Front Plant Sci. 2016;7:1417.

43. Flint-Garcia SA, Buckler ES, Tiffin P, Ersoz E, Springer NM. Heterosis is prevalent for multiple traits in diverse maize germplasm. PLoS One. 2009;4(10):e7433.

44. Melchinger AE, Utz HF, Piepho HP, Zeng ZB, Schön CC. The role of epistasis in the manifestation of heterosis: a systems-oriented approach. Genetics. 2007;177(3):1815-25

45. Yu S, Li J, Xu C, Tan Y, Gao Y, Li X, et al. Importance of epistasis as the genetic basis of heterosis in an elite rice hybrid. Proc Natl Acad Sci U S A. 1997;94:9226-31.

46. Huang X, Yang S, Gong J, Zhao Y, Feng Q, Gong H, et al. Genomic analysis of hybrid rice varieties reveals numerous superior alleles that contribute to heterosis. Nat Commun. 2015;6:6258.

47. Gao YM, Zhu J. Mapping QTLs with digenic epistasis under multiple environments and predicting heterosis based on QTL effects. Theor Appl Genet. 2007;115:325.

48. Ma X, Tang JH, Teng WT, Yan JB, Meng YJ, Li JS. Epistatic interaction is an important genetic basis of grain yield and its components in maize. Mol Breed. 2007;20:41-51.
49. Guo JJ, Chen ZL, Liu ZP, Wang BB, Song WB, Li W, et al. Identification of genetic factors affecting plant density response through QTL mapping of yield component traits in maize (Zea mays L.). Euphytica. 2011;182:409.

50. Peng B, Li Y, Wang Y, Liu C, Liu Z, Tan W, et al. QTL analysis for yield components and kernel-related traits in maize across multi-environments. Theor Appl Genet. 2011;122:1305-20.

51. Yang C, Tang D, Qu J, Zhang L, Zhang L, Chen Z, et al. Genetic mapping of QTL for the sizes of eight consecutive leaves below the tassel in maize (Zea mays L.). Theor Appl Genet. 2016;129(11):2191-209.

52. Jannink JL. Identifying quantitative trait locus by genetic background interactions in association studies. Genetics. 2007;176:553-61.

53. Chen $L, A n Y, L i ~ Y, L i C$, Shi Y, Song Y, et al. Candidate loci for yield-related traits in maize revealed by a combination of MetaQTL analysis and regional association mapping. Front Plant Sci. 2017;8:2190.

54. Austin DF, Lee M. Comparative mapping in $\mathrm{F}_{2: 3}$ and $\mathrm{F}_{6: 7}$ generations of quantitative trait loci for grain yield and yield components in maize. Theor Appl Genet. 1996;92:817-26.

55. Li YL, Niu SZ, Dong YB, Cui DQ, Wang YZ, Liu YY, et al. Identification of traitimproving quantitative trait loci for grain yield components from a dent corn inbred line in an advanced backcross $\mathrm{BC}_{2} \mathrm{~F}_{2}$ population and comparison with its $F_{2: 3}$ population in popcorn. Theor Appl Genet. 2007;115:129-40.

56. Li JZ, Zhang ZW, Li YL, Wang QL, Zhou YG. QTL consistency and metaanalysis for grain yield components in three generations in maize. Theor Appl Genet. 2011;122:771-82.

57. Hua JP, Xing YZ, Xu CG, Sun XL, Yu SB, Zhang QF. Genetic dissection of an elite rice hybrid revealed that heterozygotes are not always advantageous for performance. Genetics. 2002;162:1885-95.

58. Pan Q, Xu Y, Li K, Peng Y, Zhan W, Li W, et al. The genetic basis of plant architecture in 10 maize recombinant inbred line populations. Plant Physiol. 2017;175:858-73.

59. R Development Core Team. R: a language and environment for statistical computing. 2010.

60. Hallauer AR, Mirando FJB. Quantitative genetics in maize breeding. 2nd ed. Ames: lowa State University; 1988.

61. Knapp S, Stroup W, Ross W. Exact confidence intervals for heritability on a progeny mean basis. Crop Sci. 1985;25:192-4.

62. Bates $D$, Mächler M, Bolker B, Walker S. Fitting linear mixed-effects models using Ime4. J Stat Softw. 2015;67:1-48.

63. Chen $\mathrm{DH}$, Ronald PC. A rapid DNA minipreparation method suitable for AFLP and other PCR applications. Plant Mol Biol Report. 1999;17:53-7.

64. Fan JB, Gunderson KL, Bibikova M, Yeakley JM, Chen J, Wickham-Garcia E, et al. Illumina universal bead arrays. Methods Enzymol. 2006;410:57-73.

65. Mathias L. MapDisto: fast and efficient computation of genetic linkage maps. Mol Breed. 2012;30:1231-5.

66. Kosambi DD. The estimation of map distances from recombination values. Ann Eugenics. 1943;12:172-5.

67. Meng L, Li H, Zhang L, Wang J. QTL IciMapping: integrated software for genetic linkage map construction and quantitative trait locus mapping in biparental populations. Crop J. 2015;3(3):269-83.

68. Li H, Ye G, Wang J. A modified algorithm for the improvement of composite interval mapping. Genetics. 2007;175:361-74.

69. Li H, Ribaut JM, Li Z, Wang J. Inclusive composite interval mapping (ICIM) for digenic epistasis of quantitative traits in biparental populations. Theor Appl Genet. 2008;116:243-60.

70. Wang D, Zhu J, Li Z, Paterson A. Mapping QTLs with epistatic effects and QTL $\times$ environment interactions by mixed linear model approaches. Theor Appl Genet. 1999;99:1255-64.

71. Yang J, Hu C, Hu H, Yu R, Xia Z, Ye X, et al. QTLNetwork: mapping and visualizing genetic architecture of complex traits in experimental populations. Bioinformatics. 2008;24:721-3.

72. Yang J, Zhu J, Williams RW. Mapping the genetic architecture of complex traits in experimental populations. Bioinformatics. 2007;23:1527-36.

73. Doerge RW, Churchill GA. Permutation tests for multiple loci affecting a quantitative character. Genetics. 1996;142:285e294.

74. Rice WR. Analyzing tables of statistical tests. Evolution. 1989;43:223-5.

\section{Publisher's Note}

Springer Nature remains neutral with regard to jurisdictional claims in published maps and institutional affiliations. 